\title{
Linear differential equations and related continuous LTI systems
}

\author{
Maurizio Ciampa, Marco Franciosi, Mario Poletti \\ Dipartimento di Matematica Applicata "U. Dini" \\ Università di Pisa \\ via Buonarroti 1c, I-56126 Pisa, Italy.
}

Running head: Linear differential equations and LTI systems 


\begin{abstract}
Linear differential equations $P(D) x=Q(D) f$ with constant coefficients are considered in the framework of signal processing theory. Necessary and sufficient conditions for the existence of a continuous LTI system $\mathscr{L}$ which for every input $f$ in $L^{p}$ (resp. in $\mathscr{D}^{\prime}{ }^{p}$ ) gives an output $\mathscr{L}(f) \in \mathscr{D}^{\prime}$ such that $P(D) \mathscr{L}(f)=Q(D) f$ are stated. The complete landscape (with the exception of one pathology) of these systems is drawn. Properties and conditions related to stability and causality are analyzed.
\end{abstract}

Keywords: continuous linear time-invariant systems, continuous-time signals, distributional signals, linear differential equations.

\title{
1 Introduction
}

A signal defined on $\mathbb{R}$ is, from here on, a complex valued distribution on $\mathbb{R}$, i.e. a member of the space $\mathscr{D}^{\prime}$. This assumption includes usual signals like continuous functions and $L^{p}$ functions, as well as less usual signals like distributional derivatives of any order of continuous functions and of $L^{p}$ functions.

Let $P(D), Q(D)$ be two linear differential operators with constant coefficients in $\mathbb{C}$, such that

$$
\operatorname{deg} P(D) \geqslant 1, Q(D) \neq 0
$$

and let

$$
P(D) x=Q(D) f
$$

be the corresponding linear differential equation, in which $f=f(t)$ is a given signal and $x=x(t)$ is the unknown signal.

It is a classical result, developed in the nineteenth century and completed with the coming of the Theory of Distributions, that for every $f \in \mathscr{D}^{\prime}$ there exists at least one solution $\xi \in \mathscr{D}^{\prime}$ of Equation (1) and that the set $S(f)$ of the solutions $x \in \mathscr{D}^{\prime}$ of Equation (1) is

$$
S(f)=\left\{x=\xi+\eta \mid \eta \in C^{\infty} \text { s.t. } P(D) \eta=0\right\}
$$

Let $\mathscr{I}$ (input space) be a linear space of signals defined on $\mathbb{R}$ (i.e., a linear subspace of $\mathscr{D}^{\prime}$ ) closed under translation and equipped with a notion of convergence and limit (denoted $\mathscr{I}$-lim) for sequences, such that for every $f \in \mathscr{I}$ and every sequence $f_{k} \in \mathscr{I}$ we have

$$
f=\mathscr{I}-\lim _{k \rightarrow \infty} f_{k} \Rightarrow f=\mathscr{D}^{\prime}-\lim _{k \rightarrow \infty} f_{k}
$$

Two sorts of systems with input space $\mathscr{I}$ and output space $\mathscr{D}^{\prime}$ can be related to Equation (1) following two basic approaches to system theory.

In the first one, called behavioral approach (see [4, Chapter 3, Section 3]), the system with input space $\mathscr{I}$ and output space $\mathscr{D}^{\prime}$ related to Equation (1) is the subset $\mathscr{R}$ of $\mathscr{I} \times \mathscr{D}^{\prime}$ defined by

$$
\mathscr{R}=\left\{(f, x) \in \mathscr{I} \times \mathscr{D}^{\prime} \mid P(D) x=Q(D) f\right\}
$$

Observe that, in this approach

- $\mathscr{R}$ is linear, i.e., for every $\left(f_{1}, x_{1}\right)\left(f_{2}, x_{2}\right) \in \mathscr{R}$ and every $c \in \mathbb{C}$ we have

$$
\left(f_{1}+f_{2}, x_{1}+x_{2}\right),\left(c f_{1}, c x_{1}\right) \in \mathscr{R}
$$

- $\mathscr{R}$ is time-invariant, i.e., for every $(f(t), x(t)) \in \mathscr{R}$ and every $\tau \in \mathbb{R}$ we have

$$
(f(t-\tau), x(t-\tau)) \in \mathscr{R}
$$


- $\mathscr{R}$ is continuous, i.e., for every sequence $\left(f_{k}, x_{k}\right) \in \mathscr{R}$ and every $(f, x) \in \mathscr{I} \times \mathscr{D}^{\prime}$ such that $f=\mathscr{I}-\lim _{k \rightarrow \infty} f_{k}, x=\mathscr{D}^{\prime}-\lim _{k \rightarrow \infty} x_{k}$ it is $(f, x) \in \mathscr{R}$.

These systems have been widely studied in recent years (see [4] and [10]).

In the second approach, adopted in signal processing theory, a system with input space $\mathscr{I}$ and output space $\mathscr{D}^{\prime}$ is a map $\mathscr{L}: \mathscr{I} \rightarrow \mathscr{D}^{\prime}$ (possibly continuous, time-invariant, stable, etc...). In this approach, a system related to Equation (1) is a map

$$
\mathscr{L}: \mathscr{I} \rightarrow \mathscr{D}^{\prime}
$$

which for every input $f$ gives an output $\mathscr{L}(f)$ such that

$$
P(D) \mathscr{L}(f)=Q(D) f
$$

Every such a system will be called differential system related to $P(D), Q(D)$ and defined on $\mathscr{I}$.

Notice that in the behavioral approach Equation (1) yields an unique system $\mathscr{R}$, while the landscape of all the systems obtained in the second approach is enormous. Furthermore, whereas in the behavioral approach the unique system related to Equation (1) is linear, timeinvariant and continuous by its own definition, in the second approach the corresponding properties are not always satisfied and it arises the problem of finding those systems which are linear, time invariant (LTI for short) and continuous.

To illustrate the complexity of this problem and the information one can get from our results let us point out the following three examples.

Firstly let us consider $\mathscr{I}=L^{\infty}$ and $P(D)=D, Q(D)=1$. In this case, the differential systems related to $P(D), Q(D)$ and defined on $\mathscr{I}$ are the

$$
\mathscr{L}: L^{\infty} \rightarrow \mathscr{D}^{\prime}
$$

of the form

$$
[\mathscr{L}(f)](t)=\int_{0}^{t} f(\tau) d \tau+c(f)
$$

where $c: L^{\infty} \rightarrow \mathbb{C}$ is an arbitrary map. As we will see in Thm. 3.3 below, among them there is no continuos LTI differential system.

Secondly let us consider $\mathscr{I}=L^{1}$ and $P(D)=D+1, Q(D)=1$. In this case, the differential systems related to $P(D), Q(D)$ and defined on $\mathscr{I}$ are the

$$
\mathscr{L}: L^{1} \rightarrow \mathscr{D}^{\prime}
$$

of the form

$$
[\mathscr{L}(f)](t)=\left(e^{-t} H(t)\right) * f(t)+c(f) e^{-t}
$$

where $c: L^{1} \rightarrow \mathbb{C}$ is an arbitrary map, and $H$ is the Heaviside function. Here the symbol $\left(e^{-t} H(t)\right) * f(t)$ stands conventionally for $(g * f)(t)$, where $g(t)=e^{-t} H(t)$. In this situation, as we will see in Thm. 3.5, there exists only one continuous LTI differential system related to $P(D), Q(D)$ and defined on $L^{1}$, obtained by $c(f)=0$ for every $f$.

Thirdly let us consider $\mathscr{I}=L^{1}$ and $P(D)=D^{2}+\omega^{2}, Q(D)=1, \omega$ being a positive real number. In this case, the differential systems related to $P(D), Q(D)$ and defined on $\mathscr{I}$ are the

$$
\mathscr{L}: L^{1} \rightarrow \mathscr{D}^{\prime}
$$

of the form

$$
[\mathscr{L}(f)](t)=\left(e^{i \omega t} H(t)\right) *\left(e^{-i \omega t} H(t)\right) * f(t)+c(f) e^{i \omega t}+d(f) e^{-i \omega t}
$$


where $c: L^{1} \rightarrow \mathbb{C}, d: L^{1} \rightarrow \mathbb{C}$ are arbitrary maps. In this situation, as we will see in Thm. 3.5, there exist infinitely many continuous LTI differential systems related to $P(D), Q(D)$ and defined on $L^{1}$, obtained by $c(f)=\mu \hat{f}(\omega)$ and $d(f)=\nu \hat{f}(-\omega)$ with $\mu, \nu \in \mathbb{C}(\hat{f}$ being the Fourier transform of $f$ ).

As far as we know, no results on differential systems related to $P(D), Q(D)$ are known.

The purpose of this paper is to locate and to describe all the continuos linear timeinvariant differential systems when the input space $\mathscr{I}$ is either the usual Banach space $L^{p}$, or the subspace $\mathscr{D}_{L^{p}}^{\prime}$ of $\mathscr{D}^{\prime}$ spanned by $L^{p}$ itself and by the distributional derivatives (of any order) of its elements.

In $L^{p}$ the notion of convergence and limit (denoted $L^{p}$-lim) for sequences is the one induced by the usual norm

$$
\begin{array}{ll}
\|f\|_{p}=\left(\int_{\mathbb{R}}|f|^{p}\right)^{\frac{1}{p}} & \text { if } 1 \leqslant p<\infty \\
\|f\|_{\infty}=\operatorname{essentialsup~}_{t \in \mathbb{R}}|f(t)| & \text { if } p=\infty
\end{array}
$$

In $\mathscr{D}_{L^{p}}^{\prime}$ both the notions of weak and strong convergence and the corresponding notions of limit (denoted $w-\mathscr{D}_{L^{p}}^{\prime}$-lim and $s$ - $\mathscr{D}_{L^{p}}^{\prime}$-lim) for sequences, are considered; a system

$$
\mathscr{L}: \mathscr{D}_{L^{p}}^{\prime} \rightarrow \mathscr{D}^{\prime}
$$

will be called weakly continuous ( $w$-continuous) or strongly continuous ( $s$-continuous) if it is continuous with respect to the weak or to the strong convergence in $\mathscr{D}^{\prime}{ }^{p}$.

The leading ideas we adopt to locate and describe all the continuos LTI systems are listed below. These ideas are based on our previous results on continuous LTI systems (see $[2],[3])$ and on some generalizations for distributional spaces of the notion of convolution product (see [9]).

First idea. Let $\mathscr{L}: L^{p} \rightarrow \mathscr{D}^{\prime}$ or $\mathscr{L}: \mathscr{D}_{L^{p}}^{\prime} \rightarrow \mathscr{D}^{\prime}$ be a continuous LTI system. Then

- by $\left[3\right.$, Section II] there exists the impulse response $\Delta \in \mathscr{D}^{\prime}$ of $\mathscr{L}$, defined by

$$
\Delta=\mathscr{D}^{\prime}-\lim _{k \rightarrow \infty} \mathscr{L}\left(\varphi_{k}\right)
$$

where $\varphi_{k} \in \mathscr{D}$ is any sequence such that

$$
\mathscr{E}^{\prime}-\lim _{k \rightarrow \infty} \varphi_{k}=\delta
$$

where $\mathscr{E}^{\prime}$ is the space of distributions with compact support;

- by [3, Thms 2.1 and 2.2] it is $\Delta \in \mathscr{D}_{L^{p^{\prime}}}^{\prime}$, where as usual $1 / p+1 / p^{\prime}=1$

Second idea. Let $\mathscr{L}: L^{p} \rightarrow \mathscr{D}^{\prime}$ or $\mathscr{L}: \mathscr{D}_{L^{p}}^{\prime} \rightarrow \mathscr{D}^{\prime}$ be a continuous LTI differential system related to $P(D), Q(D)$ and let $\Delta \in \mathscr{D}_{L^{p^{\prime}}}^{\prime}$ be its impulse response. Let $\varphi_{k} \in \mathscr{D}$ be a sequence such that $\mathscr{E}^{\prime}-\lim _{k \rightarrow \infty} \varphi_{k}=\delta$. Then

$$
\begin{aligned}
& P(D)\left(\mathscr{L}\left(\varphi_{k}\right)\right)=Q(D) \varphi_{k} \\
& \mathscr{D}^{\prime}-\lim _{k \rightarrow \infty} P(D)\left(\mathscr{L}\left(\varphi_{k}\right)\right)=\mathscr{D}^{\prime}-\lim _{k \rightarrow \infty} Q(D) \varphi_{k}
\end{aligned}
$$

and, since $P(D), Q(D)$ are continuous,

$$
P(D)\left(\mathscr{D}^{\prime}-\lim _{k \rightarrow \infty} \mathscr{L}\left(\varphi_{k}\right)\right)=Q(D)\left(\mathscr{D}^{\prime}-\lim _{k \rightarrow \infty} \varphi_{k}\right)
$$


Hence by the definition of $\Delta$, we get

$$
P(D) \Delta=Q(D) \delta
$$

Thus $\Delta$ must be a fundamental solution of Equation (1) in $\mathscr{D}_{L^{p^{\prime}}}^{\prime}$, i.e. a solution of the equation

$$
\left\{\begin{array}{l}
P(D) x=Q(D) \delta \\
x \in \mathscr{D}^{\prime}{ }^{p^{\prime}}
\end{array}\right.
$$

Third idea. Let $\Xi \in \mathscr{D}_{L^{p^{\prime}}}^{\prime}$ be a solution of Equation (2). Then for every $f \in \mathscr{D}_{L^{p}}^{\prime}$ we have by the properties of convolution product

$$
P(D)(\Xi * f)=(P(D) \Xi) * f=(Q(D) \delta) * f=Q(D) f
$$

hence $\Xi * f \in S(f)$. As a consequence the LTI system

$$
\mathscr{L}_{\Xi}: \mathscr{D}_{L^{p}}^{\prime} \rightarrow \mathscr{D}^{\prime}
$$

defined, for every $f \in \mathscr{D}_{L^{p}}^{\prime}$, by $\mathscr{L}_{\Xi}(f)=\Xi * f$ is a LTI differential system related to $P(D), Q(D)$ (for the continuity and causality properties of $\mathscr{L}_{\Xi}$, see [3, Section IV]).

Here follows a brief summary of our results.

In Section 2, as asked by the first and second ideas, we consider the equation $P(D) x=$ $Q(D) \delta$ and we find necessary and sufficient conditions for the existence of solutions in $\mathscr{D}_{L^{p}}^{\prime}$; furthermore when the necessary and sufficient conditions hold we give an explicit description of these solutions (we remark that there exists an unique solution, apart from the case where the solutions are sought in $\left.\mathscr{D}_{L^{\infty}}^{\prime}\right)$.

In Section 3 we give necessary and sufficient conditions on $P(D), Q(D)$ for the existence of continuous LTI differential systems defined on $L^{p}$ or on $\mathscr{D}^{\prime} L^{p}$ associated to $P(D), Q(D)$ and, when these conditions are met, we characterize all such systems.

In Section 4 we give necessary and sufficient conditions of causality for the continuous LTI differential systems defined on $L^{p}$ or on $\mathscr{D}_{L^{p}}^{\prime}$ associated to $P(D), Q(D)$ and moreover we prove that all these systems have very good properties of "stability".

We stress that the results of this paper rest on non trivial results on uniformly almost periodic functions stated and proved in Appendix A. It is worth to mention that, although their relevance does not appear in the statement of our main theorems, uniformly almost periodic functions play an essential role in the proof of all the above mentioned theorems.

\section{Notation}

Throughout this paper we will deal with the following distributional spaces:

$$
\begin{aligned}
& \mathscr{D}^{\prime} \text { the space of distributions } \\
& \mathscr{E}^{\prime} \text { the space of distributions with compact support } \\
& \mathscr{S}^{\prime} \text { the space of tempered distributions } \\
& \mathscr{D}_{L^{p}}^{\prime} \text { the natural extension of } L^{p} \text { into } \mathscr{D}^{\prime} \\
& \dot{\mathscr{D}}_{L^{\infty}}^{\prime} \text { the space of distributions null at } \infty
\end{aligned}
$$

For a survey of the above mentioned spaces and their main properties we use in this paper we refer to [3, Section 1]. 


\section{Fundamental solutions}

Let $P(D), Q(D)$ be two linear differential operators with constant coefficients in $\mathbb{C}$, such that $\operatorname{deg} P(D) \geqslant 1, Q(D) \neq 0$ and let $P(X), Q(X) \in \mathbb{C}[X]$ be the associated polynomials. Let $\operatorname{deg} P(X)=n$ and write $P(X)$ in the form

$$
P(X)=c\left(X-s_{1}\right)^{p_{1}} \cdots\left(X-s_{r}\right)^{p_{r}}
$$

where $s_{1}=\sigma_{1}+i \omega_{1}, \ldots, s_{r}=\sigma_{r}+i \omega_{r} \in \mathbb{C}$ are pairwise distinct, and $p_{1}, \ldots, p_{r} \in \mathbb{N}$ are all $\geqslant 1$. Let $\operatorname{deg} Q(X)=m$ and write $Q(X)$ in the form

$$
Q(X)=\left(X-s_{1}\right)^{q_{1}} \cdots\left(X-s_{r}\right)^{q_{r}} Q_{1}(X)
$$

where $q_{1}, \ldots, q_{r} \in \mathbb{N}$ and $Q_{1}(X)$ is relatively prime with $P(X)$.

It is well known that $Q(X) / P(X)$ may be uniquely written in the form

$$
\frac{Q(X)}{P(X)}=\sum_{l: p_{l}>q_{l}}\left(\sum_{h=1}^{p_{l}-q_{l}} K_{l h} \frac{1}{\left(X-s_{l}\right)^{h}}\right)+R(X)
$$

where

$$
R(X)= \begin{cases}0 & \text { if } m<n \\ \sum_{h=0}^{m-n} K_{h} X^{h} & \text { if } m \geq n\end{cases}
$$

and $K_{l h}, K_{h}$ are complex numbers that satisfy

- $K_{l h} \neq 0$ for $h=p_{l}-q_{l}$;

- $K_{m-n} \neq 0$ if $m \geqslant n$.

Let $\left(\mathscr{D}_{+}^{\prime} ;+, *\right)$ be the ring of the distributions whose support is left bounded (notice that no request of a common left bound is assumed), with the usual sum "+", the convolution product "*" and the identity element given by the usual Dirac impulse $\delta=\delta(t)$ (cf. $[9$, Chapter VI, Section 5]).

In this Section we consider the differential equation

$$
P(D) x(t)=Q(D) \delta(t)
$$

finding the solutions respectively in $\mathscr{D}_{+}^{\prime}, \mathscr{D}^{\prime}, \mathscr{S}^{\prime}, \mathscr{D}_{L^{p}}^{\prime}, \dot{\mathscr{D}}_{L^{\infty}}^{\prime}$. Firstly, let us consider the differential equation

$$
\left\{\begin{array}{l}
P(D) x(t)=Q(D) \delta(t) \\
x(t) \in \mathscr{D}_{+}^{\prime}
\end{array}\right.
$$

Now, this differential equation may be written in the form

$$
\left\{\begin{array}{l}
P\left(\delta^{(1)}\right) * x(t)=Q\left(\delta^{(1)}\right) \\
x(t) \in \mathscr{D}_{+}^{\prime}
\end{array}\right.
$$

and, since $P\left(\delta^{(1)}\right)$ is invertible in $\left(\mathscr{D}_{+}^{\prime} ;+; *\right)$, taking its inverse it is immediately seen that the unique solution of Equation (3) is

$$
\xi(t)=\left[P\left(\delta^{(1)}(t)\right)\right]^{-1} * Q\left(\delta^{(1)}(t)\right)
$$

It is also well known that the use of the Laplace transform allows us to write $\xi(t)$ in the form

$$
\xi(t)=\sum_{l: p_{l}>q_{l}} \Lambda_{l}(t) e^{s_{l} t} H(t)+\Lambda(t)
$$

where 
- for every $l \in\{1, \ldots, r\}$ such that $p_{l}>q_{l}, \Lambda_{l}(t)$ is the polynomial function of degree $p_{l}-q_{l}-1$ defined by

$$
\begin{array}{r}
\Lambda_{l}(t)=\sum_{h=1}^{p_{l}-q_{l}} K_{l h} \frac{t^{h-1}}{(h-1) !} \\
\text { - } \Lambda(t)=R\left(\delta^{(1)}\right)= \begin{cases}0 & \text { if } m<n \\
\sum_{h=0}^{m-n} K_{h} \delta^{(h)}(t) & \text { if } m \geq n\end{cases}
\end{array}
$$

As an immediate consequence the solutions of

$$
\left\{\begin{array}{l}
P(D) x(t)=Q(D) \delta(t) \\
x(t) \in \mathscr{D}^{\prime}
\end{array}\right.
$$

are parametrically described by

$$
x(t)=\sum_{l: p_{l}>q_{l}} \Lambda_{l}(t) e^{s_{l} t} H(t)+\Lambda(t)+\sum_{l=1}^{r} M_{l}(t) e^{s_{l} t}
$$

where the parameters $M_{l}(t)$ are polynomial functions of degree $\leqslant p_{l}-1$. Setting

$L_{-}=\left\{l: p_{l}>q_{l}, \sigma_{l}<0\right\} \quad, \quad L_{+}=\left\{l: p_{l}>q_{l}, \sigma_{l}>0\right\} \quad, \quad L_{0}=\left\{l: p_{l}>q_{l}, \sigma_{l}=0\right\}$ and letting

$$
\begin{aligned}
\Gamma(t) & =\sum_{l \in L_{-}} \Lambda_{l}(t) e^{s_{l} t} H(t)-\sum_{l \in L_{+}} \Lambda_{l}(t) e^{s_{l} t} H(-t)+\Lambda(t) \\
\Omega(t) & =\sum_{l \in L_{0}} \Lambda_{l}(t) e^{i \omega_{l} t} H(t)
\end{aligned}
$$

it is easily seen that the solutions of Equation (5) may be parametrically described by

$$
x(t)=\Gamma(t)+\Omega(t)+\sum_{l: \sigma_{l}=0} M_{l}(t) e^{i \omega_{l} t}+\sum_{l: \sigma_{l} \neq 0} M_{l}(t) e^{s_{l} t}
$$

where the parameters $M_{l}(t)$ are still polynomial functions of degree $\leqslant p_{l}-1$; observe that

$$
\text { - } \sum_{l \in L_{-}} \Lambda_{l}(t) e^{s_{l} t} H(t)-\sum_{l \in L_{+}} \Lambda_{l}(t) e^{s_{l} t} H(-t) \in L^{1}
$$

- $\Gamma(t) \in \mathscr{D}_{L^{1}}^{\prime} \subset \dot{\mathscr{D}}_{L^{\infty}}^{\prime} \subset \mathscr{D}_{L^{\infty}}^{\prime} \subset \mathscr{S}^{\prime}$

- $\Omega(t) \in \mathscr{S}^{\prime}$, since it is a locally integrable function slowly increasing in the usual sense

- $\sum_{l: \sigma_{l}=0} M_{l}(t) e^{i \omega_{l} t} \in \mathscr{S}^{\prime}$, since it is a locally integrable function slowly increasing in the usual sense

Lemma 2.1 The solutions of

$$
\left\{\begin{array}{l}
P(D) x(t)=Q(D) \delta(t) \\
x(t) \in \mathscr{S}^{\prime}
\end{array}\right.
$$


are parametrically described by

$$
x(t)=\Gamma(t)+\Omega(t)+\sum_{l: \sigma_{l}=0} M_{l}(t) e^{i \omega_{l} t}
$$

where the parameters $M_{l}(t)$ are polynomial functions of degree $\leqslant p_{l}-1$.

Proof. By Corollary A.4 in the Appendix, the summand

$$
\sum_{l: \sigma_{l} \neq 0} M_{l}(t) e^{s_{l} t}
$$

in Equation (8) is a member of $\mathscr{S}^{\prime}$ if and only if it is null.

Lemma 2.2 The following statements are equivalent:

a) the equation

$$
\left\{\begin{array}{l}
P(D) x(t)=Q(D) \delta(t) \\
x(t) \in \mathscr{D}_{L^{\infty}}^{\prime}
\end{array}\right.
$$

has solutions

b) for every $l$ such that $\sigma_{l}=0$, it is $q_{l} \geqslant p_{l}-1$

When one of the above condition is verified, the following statements subsist:

i) $\Omega(t)=\sum_{l: \sigma_{l}=0, q_{l}=p_{l}-1} K_{l 1} e^{i \omega_{l} t} H(t)$

where $K_{l 1} \neq 0$ for every $l$.

ii) $\Omega(t) \in \mathscr{D}_{L^{\infty}}^{\prime}$

iii) the solutions of Equation (10) are parametrically described by

$$
x(t)=\Gamma(t)+\Omega(t)+\sum_{l: \sigma_{l}=0} \mu_{l} e^{i \omega_{l} t}
$$

where $\mu_{l} \in \mathbb{C}$ for every l.

Proof. a) $\Rightarrow$ b). Let $\xi(t) \in \mathscr{D}_{L^{\infty}}^{\prime}$ be such that $P(D) \xi(t)=Q(D) \delta(t)$. Since $\mathscr{D}_{L^{\infty}}^{\prime} \subset \mathscr{S}^{\prime}$, by Lemma 2.1 there exist polynomial functions $M_{l}(t)$, with degree $\leqslant p_{l}-1$, such that

$$
\xi(t)=\Gamma(t)+\Omega(t)+\sum_{l: \sigma_{l}=0} M_{l}(t) e^{i \omega_{l} t}
$$

Since $\xi(t), \Gamma(t) \in \mathscr{D}_{L^{\infty}}^{\prime}$, it is $\xi(t)-\Gamma(t) \in \mathscr{D}_{L^{\infty}}^{\prime}$ hence

$$
\sum_{l \in L_{0}} \Lambda_{l}(t) e^{i \omega_{l} t} H(t)+\sum_{l: \sigma_{l}=0} M_{l}(t) e^{i \omega_{l} t} \in \mathscr{D}_{L^{\infty}}^{\prime}
$$

Since the $\omega_{l}$ such that $\sigma_{l}=0$ are pairwise distinct, by Corollary A.6, $\Lambda_{l}(t)$ and $M_{l}(t)$ are constant functions. In particular, for every $l$ such that $\sigma_{l}=0$, if $p_{l}>q_{l}$ then it is $p_{l}-q_{l}-1=\operatorname{deg} \Lambda_{l}(t)=0$, and hence $q_{l}=p_{l}-1$. As a consequence, for every $l$ such that $\sigma_{l}=0$ it is $q_{l} \geqslant p_{l}-1$. 
b) $\Rightarrow$ i),ii). Obvious.

b) $\Rightarrow$ iii). Obviously all the

$$
x(t)=\Gamma(t)+\Omega(t)+\sum_{l: \sigma_{l}=0} \mu_{l} e^{i \omega_{l} t}
$$

(where $\mu_{l} \in \mathbb{C}$ for every $l$ ) are solutions of the equation. Let $\xi(t)$ be a solution. Since $\mathscr{D}_{L^{\infty}}^{\prime} \subset \mathscr{S}^{\prime}$, by Lemma 2.1 there exist polynomial functions $M_{l}(t)$, with degree $\leqslant p_{l}-1$, such that

$$
\xi(t)=\Gamma(t)+\Omega(t)+\sum_{l: \sigma_{l}=0} M_{l}(t) e^{i \omega_{l} t}
$$

Since $\xi(t), \Gamma(t)+\Omega(t) \in \mathscr{D}_{L^{\infty}}^{\prime}$, also

$$
\sum_{l: \sigma_{l}=0} M_{l}(t) e^{i \omega_{l} t} \in \mathscr{D}_{L^{\infty}}^{\prime}
$$

Since the $\omega_{l}$ such that $\sigma_{l}=0$ are pairwise distinct, by Corollary A.5, $M_{l}(t)$ is a constant function for every $l$.

b) $\Rightarrow$ a). Now obvious.

Lemma 2.3 The following statements are equivalent:

a) the equation

$$
\left\{\begin{array}{l}
P(D) x(t)=Q(D) \delta(t) \\
x(t) \in \dot{\mathscr{D}}_{L^{\infty}}^{\prime}
\end{array}\right.
$$

has solutions

b) for every $l$ such that $\sigma_{l}=0$, it is $q_{l} \geqslant p_{l}$

When one of the above condition is verified, the following statements subsist:

i) $\Omega(t)=0$

ii) $x(t)=\Gamma(t)$ is the only solution of Equation (11) (remember that $\Gamma(t) \in \mathscr{D}_{L^{1}}^{\prime}$ ).

Proof. a) $\Rightarrow$ b), i), ii). Let $\xi(t) \in \dot{\mathscr{D}}_{L^{\infty}}^{\prime}$ be such that $P(D) \xi(t)=Q(D) \delta(t)$. Since $\dot{\mathscr{D}}_{L^{\infty}}^{\prime} \subset \mathscr{D}_{L^{\infty}}^{\prime}$, by Lemma 2.2 there exist $\mu_{l} \in \mathbb{C}$ such that

$$
\xi(t)=\Gamma(t)+\Omega(t)+\sum_{l: \sigma_{l}=0} \mu_{l} e^{i \omega_{l} t}
$$

Since $\xi(t), \Gamma(t) \in \dot{\mathscr{D}}_{L^{\infty}}^{\prime}$, we have

$$
\Omega(t)+\sum_{l: \sigma_{l}=0} \mu_{l} e^{i \omega_{l} t} \in \dot{\mathscr{D}}_{L^{\infty}}^{\prime}
$$

Again by Lemma 2.2 we have

- for every $l$ such that $\sigma_{l}=0$, it is $q_{l} \geqslant p_{l}-1$

- $\Omega(t)=\sum_{l: \sigma_{l}=0, q_{l}=p_{l}-1} K_{l 1} e^{i \omega_{l} t} H(t)$

where $K_{l 1} \neq 0$ for every $l$. 
Hence

$$
\sum_{l: \sigma_{l}=0, q_{l}=p_{l}-1} K_{l 1} e^{i \omega_{l} t} H(t)+\sum_{l: \sigma_{l}=0} \mu_{l} e^{i \omega_{l} t} \in \dot{\mathscr{D}}_{L^{\infty}}^{\prime}
$$

As a consequence of Corollary A.7 we obtain that

- there cannot exist $l$ such that

$$
\sigma_{l}=0, q_{l}=p_{l}-1
$$

hence for every $l$ such that $\sigma_{l}=0$ it is $q_{l} \geqslant p_{l}$ (remember that, by Lemma 2.2, it is $\left.q_{l} \geqslant p_{l}-1\right)$. In particular $\Omega(t)=0$;

- $\mu_{l}=0$ for every $l$ hence, in particular, $\Gamma(t)$ is the only solution of Equation (11).

b) $\Rightarrow$ a). Obviously we have

$$
P(D)(\Gamma(t)+\Omega(t))=Q(D)(\delta(t))
$$

Hence, since by assumption it is $\Omega(t)=0$, and by construction it is $\Gamma(t) \in \dot{\mathscr{D}}_{L^{\infty}}^{\prime}$, we conclude that $\Gamma(t)$ is a solution of Equation (11)

Lemma 2.4 Let $1 \leqslant p<\infty$. The following statements are equivalent:

a) the equation

$$
\left\{\begin{array}{l}
P(D) x(t)=Q(D) \delta(t) \\
x(t) \in \mathscr{D}^{\prime} L^{p}
\end{array}\right.
$$

has solutions

b) for every $l$ such that $\sigma_{l}=0$, it is $q_{l} \geqslant p_{l}$

When one of the above condition is verified, the following statements subsist:

i) $\Omega(t)=0$

ii) $x(t)=\Gamma(t)$ is the only solution of Equation (12) (remember that $\Gamma(t) \in \mathscr{D}_{L^{1}}^{\prime}$ )

Proof. a) $\Rightarrow$ b),i),ii). Since $\mathscr{D}_{L^{p}}^{\prime} \subset \dot{\mathscr{D}}_{L^{\infty}}^{\prime}$, the equation

$$
\left\{\begin{array}{l}
P(D) x(t)=Q(D) \delta(t) \\
x(t) \in \dot{\mathscr{D}}_{L^{\infty}}^{\prime}
\end{array}\right.
$$

has solutions. Hence the statements follow by Lemma 2.3 .

b) $\Rightarrow$ a). By Lemma 2.3, the equation

$$
\left\{\begin{array}{l}
P(D) x(t)=Q(D) \delta(t) \\
x(t) \in \dot{\mathscr{D}}_{L^{\infty}}^{\prime}
\end{array}\right.
$$

has solutions, and $x(t)=\Gamma(t)$ is the only solution. Since $\Gamma(t) \in \mathscr{D}_{L^{1}}^{\prime} \subseteq \mathscr{D}_{L^{p}}^{\prime}$, the statement is proved. 


\section{The landscape of continuos LTI differential systems de- fined on $L^{p}$ and on $\mathscr{D}_{L^{p}}^{\prime}$}

We can now give a complete description (with the exception of one pathology) of the landscape of continuous LTI differential systems

$$
\mathscr{L}: L^{p} \rightarrow \mathscr{D}^{\prime} \quad \mathscr{L}: \mathscr{D}^{\prime}{ }^{p} \rightarrow \mathscr{D}^{\prime}
$$

related to $P(D), Q(D)$. Unfortunately our picture is not so short as we hoped, but in our opinion now the results should be particularly clear and readable, such as their corresponding proofs.

So, as in the previous section, let $P(X), Q(X) \in \mathbb{C}[X]$ - the polynomials associated to $P(D), Q(D)$ - be written as

$$
\begin{gathered}
P(X)=c\left(X-s_{1}\right)^{p_{1}} \cdots\left(X-s_{r}\right)^{p_{r}} \\
Q(X)=\left(X-s_{1}\right)^{q_{1}} \cdots\left(X-s_{r}\right)^{q_{r}} Q_{1}(X)
\end{gathered}
$$

where $s_{1}=\sigma_{1}+i \omega_{1}, \ldots, s_{r}=\sigma_{r}+i \omega_{r} \in \mathbb{C}$, and let $\Gamma(t) \in \mathscr{D}_{L^{1}}^{\prime}, \Omega(t) \in \mathscr{S}^{\prime}$, be the distributions defined in Equations (6), (7). Finally remember that if $1<p<q<\infty$ then there are the following inclusions

$$
\mathscr{D}_{L^{1}}^{\prime} \subset \mathscr{D}_{L^{p}}^{\prime} \subset \mathscr{D}_{L^{q}}^{\prime} \subset \dot{\mathscr{D}}_{L^{\infty}}^{\prime} \subset \mathscr{D}_{L^{\infty}}^{\prime} \subset \mathscr{S}^{\prime}
$$

As it is usual in studying $L^{p}$ and $\mathscr{D}_{L^{p}}^{\prime}$ we will distinguish between the case $1<p<\infty$ and the cases $p=1, p=\infty$. Our first result is the following:

Theorem 3.1 Let $1<p<\infty$. The following statements are equivalent:

i) the rational function $Q(s) / P(s)$ has no pole on the complex imaginary axis, i.e. for every $l$ such that $\sigma_{l}=0$, it is $q_{l} \geqslant p_{l}$

ii) there exists a w-continuous LTI differential system

$$
\mathscr{L}: \mathscr{D}_{L^{p}}^{\prime} \rightarrow \mathscr{D}^{\prime}
$$

related to $P(D), Q(D)$

iii) there exists a s-continuous LTI differential system

$$
\mathscr{L}: \mathscr{D}_{L^{p}}^{\prime} \rightarrow \mathscr{D}^{\prime}
$$

related to $P(D), Q(D)$

iv) there exists a continuous LTI differential system

$$
\mathscr{L}: L^{p} \rightarrow \mathscr{D}^{\prime}
$$

related to $P(D), Q(D)$

If one of the above equivalent conditions is verified then the following statements hold:

a) $\mathscr{L}_{\Gamma}: \mathscr{D}_{L^{p}}^{\prime} \rightarrow \mathscr{D}^{\prime}$ is the only $w$-continuous (resp. s-continuous) LTI differential system defined on $\mathscr{D}_{L^{p}}^{\prime}$, related to $P(D), Q(D)$

b) the restriction of $\mathscr{L}_{\Gamma}$ to $L^{p}$ is the only continuous LTI differential system defined on $L^{p}$, related to $P(D), Q(D)$ 
c) for every $f \in \mathscr{D}_{L^{p}}^{\prime}, \mathscr{L}_{\Gamma}(f)$ is the only distribution null at $\infty$ (i.e. a member of $\dot{\mathscr{D}}_{L^{\infty}}^{\prime}$ ) which solves the equation

$$
P(D) x=Q(D) f
$$

Proof. ii $\Rightarrow$ i), iii) $\Rightarrow$ i), iv) $\Rightarrow$ i). Let $\mathscr{L}$ be a system as in ii), iii), iv). As pointed out in Section 1, there exists the impulse response $\Delta \in \mathscr{D}^{\prime}$ of $\mathscr{L}$, and we have $\Delta \in \mathscr{D}_{L^{p^{\prime}}}^{\prime}$ (see First idea), moreover $P(D) \Delta=Q(D) \delta$ (see Second idea). Hence the equation

$$
\left\{\begin{array}{l}
P(D) x(t)=Q(D) \delta(t) \\
x(t) \in \mathscr{D}^{\prime}{ }^{p^{\prime}}
\end{array}\right.
$$

has at least one solution (namely $\Delta$ ). As a consequence, by Lemma 2.4, for every $l$ such that $\sigma_{l}=0$, it is $q_{l} \geqslant p_{l}$.

i) $\Rightarrow$ a). Let us firstly consider the system

$$
\begin{array}{ccc}
\mathscr{L}_{\Gamma}: \mathscr{D}^{\prime} L^{p} & \longrightarrow & \mathscr{D}^{\prime} \\
f & \mapsto & \Gamma * f
\end{array}
$$

Since $\Gamma(t) \in \mathscr{D}_{L^{1}}^{\prime} \subset \mathscr{D}_{L^{p^{\prime}}}^{\prime}$, by [3, Thms 4.2 and 4.3], $\mathscr{L}_{\Gamma}$ is a weakly (resp. strongly) continuous LTI system. By Lemma 2.4 it is $P(D) \Gamma=Q(D) \delta$; hence, as pointed out in Section 1 (see Third idea), for every $f \in \mathscr{D}_{L^{p}}^{\prime}$ we have

$$
P(D)(\Gamma * f)=(P(D) \Gamma) * f=(Q(D) \delta) * f=Q(D) f
$$

Thus $\mathscr{L}_{\Gamma}$ is also a differential system related to $P(D), Q(D)$.

Now let $\mathscr{L}: \mathscr{D}_{L^{p}}^{\prime} \rightarrow \mathscr{D}^{\prime}$ be a weakly (resp. strongly) continuous LTI differential system related to $P(D), Q(D)$, and let $\Delta$ be its impulse response. As above we obtain that $\Delta$ is a solution of the equation

$$
\left\{\begin{array}{l}
P(D) x(t)=Q(D) \delta(t) \\
x(t) \in \mathscr{D}^{\prime} L^{p^{\prime}}
\end{array}\right.
$$

and then by Lemma 2.4 we have $\Delta=\Gamma$. By [3, Thm. 5.3] we obtain $\mathscr{L}=\mathscr{L}_{\Gamma}$.

i) $\Rightarrow$ b). First note that the restriction of $\mathscr{L}_{\Gamma}$ to $L^{p}$ is a continuous differential system related to $P(D), Q(D)$.

Now let $\mathscr{L}: L^{p} \rightarrow \mathscr{D}^{\prime}$ be a continuous LTI differential system related to $P(D), Q(D)$, and let $\Delta \in \mathscr{D}^{\prime}$ be its impulse response. As in the proof of i) $\Rightarrow$ a), we obtain $\Delta=\Gamma$. By [3, Thm. 5.1] $\mathscr{L}$ is the restriction of $\mathscr{L}_{\Gamma}$ to $L^{p}$.

i) $\Rightarrow \mathrm{c})$. Since $\Gamma \in \mathscr{D}_{L^{1}}^{\prime}, f \in \mathscr{D}_{L^{p}}^{\prime}$ and $1<p<\infty$, by [3, Thm. 4.1] we obtain $\mathscr{L}_{\Gamma}(f) \in$ $\mathscr{D}_{L^{p}}^{\prime} \subset \dot{\mathscr{D}}_{L^{\infty}}^{\prime}$. Now let $g(t) \in S(f) \cap \dot{\mathscr{D}}_{L^{\infty}}^{\prime}$; we can write

$$
g(t)=\mathscr{L}_{\Gamma}(f)+\sum_{l: \sigma_{l}=0} M_{l}(t) e^{i \omega_{l} t}+\sum_{l: \sigma_{l} \neq 0} M_{l}(t) e^{s_{l} t}
$$

where $M_{l}(t)$ are polynomials (see Equation (8)). Thus since the first and second summands $\in \mathscr{S}^{\prime}$, then

$$
\sum_{l: \sigma_{l} \neq 0} M_{l}(t) e^{s_{l} t} \in \mathscr{S}^{\prime}
$$

and hence, by Corollary A.4, we obtain that for every $l$ such that $\sigma_{l} \neq 0$ it is $M_{l}(t)=0$. As a consequence

$$
\sum_{l: \sigma_{l}=0} M_{l}(t) e^{i \omega_{l} t}
$$


is a distribution null at $\infty$. By Corollary A.5, for every $l$ such that $\sigma_{l}=0$, there exists $K_{l} \in \mathbb{C}$ such that $M_{l}(t)=K_{l}$. As a consequence we have that

$$
\sum_{l: \sigma_{l}=0} K_{l} e^{i \omega_{l} t}
$$

is a distribution null at $\infty$; by Corollary A.2, for every $l$ such that $\sigma_{l}=0$ we have $M_{l}(t)=$ $K_{l}=0$. Hence $g(t)=\mathscr{L}_{\Gamma}(f)$. To prove that i) $\Rightarrow$ ii), i) $\Rightarrow$ iii), i) $\Rightarrow$ iv), merely observe that a) $\Rightarrow$ ii), a) $\Rightarrow$ iii), b) $\Rightarrow$ iv).

Now we analyze the case $p=\infty$.

Theorem 3.2 Let $p=\infty$. The following statements are equivalent:

i) the rational function $Q(s) / P(s)$ has no pole on the complex imaginary axis, i.e. for every $l$ such that $\sigma_{l}=0$, it is $q_{l} \geqslant p_{l}$

ii) there exists a w-continuous LTI differential system

$$
\mathscr{L}: \mathscr{D}^{\prime}{ }^{\infty} \rightarrow \mathscr{D}^{\prime}
$$

related to $P(D), Q(D)$

If one of the above equivalent conditions is verified then the following statements hold:

a) $\mathscr{L}_{\Gamma}: \mathscr{D}_{L^{\infty}}^{\prime} \rightarrow \mathscr{D}^{\prime}$ is the only $w$-continuous LTI differential system defined on $\mathscr{D}_{L^{\infty}}^{\prime}$, related to $P(D), Q(D)$

b) for every $f \in \mathscr{D}^{\prime}{ }^{\infty}$,

$$
\mathscr{L}_{\Gamma}(f)+\left\langle e^{i \omega_{l} t}: l \text { such that } \sigma_{l}=0\right\rangle
$$

is the set of all the bounded distributions (i.e. members of $\mathscr{D}_{L^{\infty}}$ ) which solve the equation

$$
P(D) x=Q(D) f
$$

Proof. ii $\Rightarrow$ i). Let $\mathscr{L}$ be a system as in ii), and let $\Delta \in \mathscr{D}^{\prime}$ be its impulse response. As in the Proof of Theorem 3.1, we obtain that $\Delta$ is a solution of the equation

$$
\left\{\begin{array}{l}
P(D) x(t)=Q(D) \delta(t) \\
x(t) \in \mathscr{D}^{\prime} L^{1}
\end{array}\right.
$$

As a consequence, by Lemma 2.4, for every $l$ such that $\sigma_{l}=0$, it is $q_{l} \geqslant p_{l}$.

i) $\Rightarrow$ a). Let $\mathscr{L}: \mathscr{D}^{\prime}{ }^{\infty} \rightarrow \mathscr{D}^{\prime}$ be a weakly continuous LTI differential system related to $P(D), Q(D)$, and let $\Delta \in \mathscr{D}^{\prime}$ be its impulse response. As above we obtain that $\Delta$ is a solution of

$$
\left\{\begin{array}{l}
P(D) x(t)=Q(D) \delta(t) \\
x(t) \in \mathscr{D}^{\prime} L^{1}
\end{array}\right.
$$

As a consequence, by Lemma 2.4 we have $\Delta=\Gamma$. By $\left[3\right.$, Thm. 5.6] we obtain $\mathscr{L}=\mathscr{L}_{\Gamma}$. To show the existence, as in the proof of i) $\Rightarrow$ a) of Theorem 3.1, we obtain that $\mathscr{L}_{\Gamma}: \mathscr{D}^{\prime} L^{\infty} \rightarrow \mathscr{D}^{\prime}$ is a weakly continuous LTI differential system related to $P(D), Q(D)$.

i) $\Rightarrow$ b) Same argument of the Proof of i) $\Rightarrow$ c) of Theorem 3.1.

To prove that i) $\Rightarrow$ ii), merely observe that a) $\Rightarrow$ ii).

Theorem 3.3 Let $p=\infty$. The following statements are equivalent:

i) the rational function $Q(s) / P(s)$ has no pole on the complex imaginary axis, i.e. for every $l$ such that $\sigma_{l}=0$, it is $q_{l} \geqslant p_{l}$ 
ii) there exists a s-continuous LTI differential system

$$
\mathscr{L}: \mathscr{D}^{\prime}{ }^{\infty} \rightarrow \mathscr{D}^{\prime}
$$

related to $P(D), Q(D)$

iii) there exists a continuous LTI differential system

$$
\mathscr{L}: L^{\infty} \rightarrow \mathscr{D}^{\prime}
$$

related to $P(D), Q(D)$

If one of the above equivalent conditions is verified then the following statements hold:

a) $\mathscr{L}_{\Gamma}: \mathscr{D}_{L^{\infty}}^{\prime} \rightarrow \mathscr{D}^{\prime}$ is a strongly continuous LTI differential system defined on $\mathscr{D}_{L^{\infty}}^{\prime}$, related to $P(D), Q(D)$

(the pathology of $\mathscr{D}_{L^{\infty}}^{\prime}$ doesn't allow us to say that $\mathscr{L}=\mathscr{L}_{\Gamma}$ )

b) the restriction of $\mathscr{L}_{\Gamma}$ to $L^{\infty}$ is a continuous LTI differential system defined on $L^{\infty}$, related to $P(D), Q(D)$

(the pathology of $L^{\infty}$ doesn't allow us to say that $\mathscr{L}$ is the restriction of $\mathscr{L}_{\Gamma}$ to $L^{\infty}$ )

c) let $\mathscr{L}: \mathscr{D}_{L^{\infty}}^{\prime} \rightarrow \mathscr{D}^{\prime}$ be a s-continuous LTI differential system related to $P(D), Q(D)$, then for every $f \in \dot{\mathscr{D}}_{L^{\infty}}^{\prime}$ we have

$$
\mathscr{L}(f)=\mathscr{L}_{\Gamma}(f)=\Gamma * f
$$

d) let $\mathscr{L}: L^{\infty} \rightarrow \mathscr{D}^{\prime}$ be a continuous LTI differential system related to $P(D), Q(D)$, then for every $f \in \mathscr{C}_{\circ}$ (where $\mathscr{C}_{\circ}$ is the space of the $C^{0}$ functions null at infinity in the usual sense) we have

$$
\mathscr{L}(f)=\mathscr{L}_{\Gamma}(f)=\Gamma * f
$$

Proof. ii $\Rightarrow$ i). Same argument of ii) $\Rightarrow$ i) in the Proof of Theorem 3.2.

i) $\Rightarrow$ a). Already proved in the Proof of Theorem 3.2.

i) $\Rightarrow$ b). Merely observe that i) $\Rightarrow a$ ), and that a) $\Rightarrow b$ ).

i) $\Rightarrow$ c),i $\Rightarrow$ d). Let $\Delta$ be the impulse response of $\mathscr{L}$. As in i) $\Rightarrow$ a) of Theorem 3.2 we obtain $\Delta=\Gamma$. Hence, the statements follow by $[3$, Thms. $5.2,5.7]$

i) $\Rightarrow$ ii), i $\Rightarrow$ iii). Merely observe that a) $\Rightarrow$ ii), b) $\Rightarrow$ iii).

We remark that the analysis of the case $p=\infty$ does not give a complete description of the corresponding continuous LTI differential systems since on one hand it shows the typical pathology of strong convergence, while on the other hand it gives no evidence of the meaning of $\mathscr{L}_{\Gamma}(f)$.

At last we consider the case $p=1$.

Theorem 3.4 Let $p=1$. The following statements are equivalent:

i) the rational function $Q(s) / P(s)$ has no pole on the complex imaginary axis, i.e. for every $l$ such that $\sigma_{l}=0$, it is $q_{l} \geqslant p_{l}$

ii) there exists a w-continuous LTI differential system

$$
\mathscr{L}: \mathscr{D}_{L^{1}}^{\prime} \rightarrow \mathscr{D}^{\prime}
$$

related to $P(D), Q(D)$

If one of the above equivalent conditions is verified then the following statements hold: 
a) $\mathscr{L}_{\Gamma}: \mathscr{D}_{L^{1}}^{\prime} \rightarrow \mathscr{D}^{\prime}$ is the only weakly continuous LTI differential system defined on $\mathscr{D}_{L^{1}}^{\prime}$, related to $P(D), Q(D)$

b) for every $f \in \mathscr{D}_{L^{1}}^{\prime}, \mathscr{L}_{\Gamma}(f)$ is the only distribution null at $\infty$ which solves the equation

$$
P(D) x=Q(D) f
$$

Proof. ii) $\Rightarrow$ i). Let $\mathscr{L}$ be as in ii), and let $\Delta \in \mathscr{D}^{\prime}$ be its impulse response. By [3, Thm. 2.3] we have $\Delta \in \dot{\mathscr{D}}_{L^{\infty}}^{\prime}$, hence the equation

$$
\left\{\begin{array}{l}
P(D) x(t)=Q(D) \delta(t) \\
x(t) \in \dot{\mathscr{D}}_{L^{\infty}}^{\prime}
\end{array}\right.
$$

has at least one solution (namely $\Delta$ ). As a consequence, by Lemma 2.3 , for every $l$ such that $\sigma_{l}=0$, it is $q_{l} \geqslant p_{l}$.

i) $\Rightarrow$ a). Let $\mathscr{L}: \mathscr{D}_{L^{1}}^{\prime} \rightarrow \mathscr{D}^{\prime}$ be a weakly continuous LTI differential system related to $P(D), Q(D)$, and let $\Delta \in \mathscr{D}^{\prime}$ be its impulse response. As above, we obtain that $\Delta$ is a solution of

$$
\left\{\begin{array}{l}
P(D) x(t)=Q(D) \delta(t) \\
x(t) \in \dot{\mathscr{D}}_{L^{\infty}}^{\prime}
\end{array}\right.
$$

hence, by Lemma 2.3 we have $\Delta=\Gamma$. By [3, Thm. 5.4] we obtain $\mathscr{L}=\mathscr{L}_{\Gamma}$. As in the proof of statement $\mathrm{i} \Rightarrow \mathrm{b}$ ) of Theorem 3.1 , we obtain that $\mathscr{L}_{\Gamma}: \mathscr{D}_{L^{1}}^{\prime} \rightarrow \mathscr{D}^{\prime}$ is a $w$-continuous LTI differential system related to $P(D), Q(D)$.

i) $\Rightarrow$ b). Same argument of i) $\Rightarrow c$ ) in the Proof of Theorem 3.1.

i) $\Rightarrow$ ii). Merely observe that i) $\Rightarrow$ a) and that a) $\Rightarrow$ ii).

Studying continuous LTI differential systems defined on $L^{1}$ and $s$-continuous LTI differential systems defined on $\mathscr{D}_{L^{1}}^{\prime}$ is quite subtle and needs the notion of distributions null at $-\infty$.

Let $f(t) \in \mathscr{D}^{\prime} ; f(t)$ will be called a distribution null at $-\infty$ if it verifies one of the following equivalent conditions:

- $\mathscr{D}^{\prime}-\lim _{\tau \rightarrow+\infty} f(t-\tau)=0$

- for every $\varphi \in \mathscr{D}$, it is $\lim _{t \rightarrow-\infty}(f * \varphi)(t)=0$

(the proof of the equivalence is straightforward).

Obviously

- every locally integrable function $f(t)$, null at $-\infty$ in the usual sense, is a distribution null at $-\infty$

- every $f(t) \in \dot{\mathscr{D}}_{L^{\infty}}^{\prime}$, is a distribution null at $-\infty$

- every $f(t) \in \mathscr{D}_{L^{p}}^{\prime}$, with $1 \leqslant p<\infty$, is a distribution null at $-\infty$

Handling this concept, a little attention must be used: there are functions non null at $-\infty$ in the usual sense, which are distributions null al $-\infty$; for instance $f(t)=e^{i t^{2}}$ (for the non trivial proof see [9, Chapter VII,Section 5]).

Remark 3.1 Let $f(t) \in \mathscr{D}^{\prime}{ }^{1}$, and let $\omega_{0} \in \mathbb{R}$. Since $f(t) \in \mathscr{S}^{\prime}$, we may consider the Fourier Transform $\hat{f}(\omega)$ of $f(t) .{ }^{1}$ The following statements hold

\footnotetext{
${ }^{1}$ For every $f(t) \in L^{1}$, the definition of Fourier Transform we adopt is the following: $\hat{f}(\omega)=\int_{\mathbb{R}} e^{-i \omega t} f(t) \mathrm{d} t$
} 
a) $\hat{f}(\omega) \in C^{0}$

b) $e^{i \omega_{0} t} * f(t)=\hat{f}\left(\omega_{0}\right) e^{i \omega_{0} t}$

Proof. Writing $f(t)$ in the form

$$
f(t)=\sum_{h=0}^{\mu} f_{h}^{(h)}(t)
$$

with $f_{h}(t) \in L^{1}$ for every $h$, we obtain

$$
\hat{f}(\omega)=\sum_{h=0}^{\mu}(i \omega)^{h} \hat{f}_{h}(\omega)
$$

a) Since $\hat{f}_{h}(\omega) \in C^{0}$ for every $h$, also $\hat{f}(\omega) \in C^{0}$.

b) By $[3$, Section III] we have

$$
\begin{aligned}
e^{i \omega_{0} t} * f(t) & =\sum_{h=0}^{\mu}\left(D^{h} e^{i \omega_{0} t}\right) * f_{h}(t)=\sum_{h=0}^{\mu}\left(i \omega_{0}\right)^{h} e^{i \omega_{0} t} * f_{h}(t)= \\
& =\sum_{h=0}^{\mu}\left(i \omega_{0}\right)^{h} \int f_{h}(\tau) e^{i \omega_{0}(t-\tau)} \mathrm{d} \tau=e^{i \omega_{0} t} \sum_{h=0}^{\mu}\left(i \omega_{0}\right)^{h} \hat{f}_{h}\left(\omega_{0}\right)=\hat{f}\left(\omega_{0}\right) e^{i \omega_{0} t}
\end{aligned}
$$

Remark 3.2 Let $\omega_{1}, \ldots, \omega_{\nu} \in \mathbb{R}, c_{1}, \ldots, c_{\nu} \in \mathbb{C}, f(t) \in \mathscr{D}_{L^{1}}^{\prime}$. Then

$$
\left(\sum_{l=1}^{\nu} c_{l} e^{i \omega_{l} t} H(t)\right) * f(t)
$$

is a distribution null at $-\infty$.

Proof. Let $\omega_{0} \in \mathbb{R}, g(t) \in L^{1}$, and let

$$
G(t)=\left(e^{i \omega_{0} t} H(t)\right) * g(t)
$$

Since

$$
G(t)=\int g(\tau) e^{i \omega_{0}(t-\tau)} H(t-\tau) \mathrm{d} \tau=e^{i \omega_{0} t} \int_{-\infty}^{t} g(\tau) e^{-i \omega_{0} \tau} \mathrm{d} \tau
$$

then $G(t)$ is a continuous function, bounded and null at $-\infty$ in the usual sense. As a consequence, $G(t)$ is a distribution null at $-\infty$.

Let $h \in \mathbb{N}$; by $[3$, Section III] we have

$$
\left(e^{i \omega_{0} t} H(t)\right) * g^{(h)}(t)=G^{(h)}(t)
$$

hence, for every $\varphi \in \mathscr{D}$, we have

$$
\lim _{t \rightarrow-\infty}\left(G^{(h)} * \varphi\right)(t)=\lim _{t \rightarrow-\infty}\left(G * \varphi^{(h)}\right)(t)=0
$$

As a consequence, $\left(e^{i \omega_{0} t} H(t)\right) * g^{(h)}(t)$ is a distribution null at $-\infty$.

Since $f(t)$ may be written in the form $f(t)=\sum_{h=0}^{\mu} f_{h}^{(h)}(t)$ with $f_{h}(t) \in L^{1}$ for every $h$, the statement follows by the above argument.

With the help of the notion of distributions null at $-\infty$ and the above results we obtain the following theorem. 
Theorem 3.5 Let $p=1$. The following statements are equivalent:

i) the rational function $Q(s) / P(s)$ has no pole of order $>1$ on the complex imaginary axis, i.e. for every $l$ such that $\sigma_{l}=0$, it is $q_{l} \geqslant p_{l}-1$

ii) there exists a s-continuous LTI differential system

$$
\mathscr{L}: \mathscr{D}_{L^{1}}^{\prime} \rightarrow \mathscr{D}^{\prime}
$$

related to $P(D), Q(D)$

iii) there exists a continuous LTI differential system

$$
\mathscr{L}: L^{1} \rightarrow \mathscr{D}^{\prime}
$$

related to $P(D), Q(D)$

If one of the above conditions is verified then

a) $\Omega(t)=\sum_{l: \sigma_{l}=0, q_{l}=p_{l}-1} K_{l 1} e^{i \omega_{l} t} H(t) \in L^{\infty}$, and $K_{l 1} \neq 0$ for every $l$.

Furthermore for every family of complex numbers

$$
\mu=\left(\mu_{l} \in \mathbb{C}: l \text { such that } \sigma_{l}=0\right)
$$

let

$$
\Phi_{\mu}(t)=\Gamma(t)+\Omega(t)+\sum_{l: \sigma_{l}=0} \mu_{l} e^{i \omega_{l} t}
$$

Then we have:

b) $\Phi_{\mu}(t) \in \mathscr{D}^{\prime}{ }^{\infty}$ for every $\mu$

c) the family (depending on $\mu$ )

$$
\mathscr{L}_{\mu}=\mathscr{L}_{\Phi_{\mu}}: \mathscr{D}_{L^{1}}^{\prime} \rightarrow \mathscr{D}^{\prime}
$$

is the family of all s-continuous LTI differential systems defined on $\mathscr{D}_{L^{1}}^{\prime}$, related to $P(D), Q(D)$; in particular

$$
\mathscr{L}_{0}=\mathscr{L}_{(\Gamma+\Omega)}: \mathscr{D}_{L^{1}}^{\prime} \rightarrow \mathscr{D}^{\prime}
$$

is a s-continuous LTI differential system related to $P(D), Q(D)$

d) the family (depending on $\mu$ ) of the restrictions of the $\mathscr{L}_{\mu}$ to $L^{1}$ is the family of all continuous LTI differential systems defined on $L^{1}$, related to $P(D), Q(D)$

e) for every $\mu$ and every $f(t) \in \mathscr{D}_{L^{1}}^{\prime}$ it is

$$
\mathscr{L}_{\mu}(f(t))=\mathscr{L}_{(\Gamma+\Omega)}(f(t))+\sum_{l: \sigma_{l}=0} \mu_{l} \hat{f}\left(\omega_{l}\right) e^{i \omega_{l} t}
$$

f) for every $f \in \mathscr{D}_{L^{1}}^{\prime}, \mathscr{L}_{0}(f)=\mathscr{L}_{(\Gamma+\Omega)}(f)$ is the only distribution null at $-\infty$ and bounded which solve the equation

$$
P(D) x=Q(D) f
$$


Observe that the families of systems described in c) (respectively $d)$ ) reduces to one element if and only if $P(s)$ has no roots on the imaginary axis.

Proof. ii) $\Rightarrow$ i), iii) $\Rightarrow$ i). Let $\mathscr{L}$ be as in ii) or as in iii), and let $\Delta \in \mathscr{D}^{\prime}$ be its impulse response. As in the Proof of Theorem 3.1, we obtain that $\Delta$ is a solution of the equation

$$
\left\{\begin{array}{l}
P(D) x(t)=Q(D) \delta(t) \\
x(t) \in \mathscr{D}^{\prime} L^{\infty}
\end{array}\right.
$$

Hence, by Lemma 2.2, for every $l$ such that $\sigma_{l}=0$, it is $q_{l} \geqslant p_{l}-1$.

i) $\Rightarrow$ a), i $\Rightarrow$ b). See statements i), ii) of Lemma 2.2.

i) $\Rightarrow$ c). Let $\mathscr{L}: \mathscr{D}_{L^{1}}^{\prime} \rightarrow \mathscr{D}^{\prime}$ be a $s$-continuous LTI differential system related to $P(D), Q(D)$, and let $\Delta \in \mathscr{D}^{\prime}$ be its impulse response. As above we obtain that $\Delta$ is a solution of the equation

$$
\left\{\begin{array}{l}
P(D) x(t)=Q(D) \delta(t) \\
x(t) \in \mathscr{D}^{\prime} L^{\infty}
\end{array}\right.
$$

As a consequence, by Lemma 2.2 , there exists $\mu$ such that $\Delta=\Phi_{\mu}$. By [3, Thm. 5.5], we obtain $\mathscr{L}=\mathscr{L}_{\mu}$.

As in i) $\Rightarrow$ a) in the Proof of Theorem 3.1, we obtain that $\mathscr{L}_{\mu}: \mathscr{D}_{L^{1}}^{\prime} \rightarrow \mathscr{D}^{\prime}$ is a $s$-continuous LTI differential system related to $P(D), Q(D)$.

i) $\Rightarrow$ d). Same argument of i) $\Rightarrow$ b) in the Proof of Theorem 3.1.

i) $\Rightarrow$ e). Follows by Remark 3.1.

i) $\Rightarrow$ f). Obviously $\mathscr{L}_{(\Gamma+\Omega)}(f)$ is a bounded distribution. Moreover by Remark 3.2 it is a distribution null at $-\infty$.

Now let $g(t) \in S(f)$ be a distribution null at $-\infty$ and bounded. There exist polynomials $M_{l}(t)$ such that

$$
g(t)=\mathscr{L}_{(\Gamma+\Omega)}(f(t))+\sum_{l: \sigma_{l}=0} M_{l}(t) e^{i \omega_{l} t}+\sum_{l: \sigma_{l} \neq 0} M_{l}(t) e^{i s_{l} t}
$$

Since $g \in \mathscr{S}^{\prime}$ and

$$
\mathscr{L}_{(\Gamma+\Omega)}(f(t))+\sum_{l: \sigma_{l}=0} M_{l}(t) e^{i \omega_{l} t} \in \mathscr{S}^{\prime}
$$

then

$$
\sum_{l: \sigma_{l} \neq 0} M_{l}(t) e^{i s_{l} t} \in \mathscr{S}^{\prime}
$$

hence, by Corollary A. 4 , for every $l$ such that $\sigma_{l} \neq 0$, we have $M_{l}(t)=0$. As a consequence

$$
\sum_{l: \sigma_{l}=0} M_{l}(t) e^{i \omega_{l} t}=g(t)-\mathscr{L}_{(\Gamma+\Omega)}(f(t))
$$

is a distribution null at $-\infty$ and bounded. By Corollary A.6, for every $l$ such that $\sigma_{l}=0$ there exist $K_{l} \in \mathbb{C}$ such that $M_{l}(t)=K_{l}$. As a consequence

$$
\sum_{l: \sigma_{l}=0} K_{l} e^{i \omega_{l} t}
$$

is a distribution null at $-\infty$; hence, by Corollary A.2, for every $l$ such that $\sigma_{l}=0$ we have $M_{l}(t)=K_{l}=0$

i) $\Rightarrow$ ii), i $\Rightarrow$ iii). Merely observe that i) $\Rightarrow c)$, i) $\Rightarrow$ d), and that $c) \Rightarrow$ ii), d) $\Rightarrow$ iii). 


\section{Properties and conditions related to stability and causality}

In the above section we have just seen that every continuous LTI differential system $\mathscr{L}$ : $L^{p} \rightarrow \mathscr{D}^{\prime}, \mathscr{L}: \mathscr{D}_{L^{p}}^{\prime} \rightarrow \mathscr{D}^{\prime}$, can be identified with systems of the form $\mathscr{L}_{\Gamma}$ or $\mathscr{L}_{\mu}$.

In this section we analyze some interesting properties of these $\mathscr{L}_{\Gamma}$ and $\mathscr{L}_{\mu}$.

We recall that a system $\mathscr{L}: \mathscr{I} \rightarrow \mathscr{D}^{\prime}$ is said to be causal if for every $t_{0} \in \mathbb{R}$ and for every $f, g \in \mathscr{I}$ with $\operatorname{supp}(f-g) \subset\left[t_{0},+\infty\right)$, it is $\operatorname{supp}(\mathscr{L}(f)-\mathscr{L}(g)) \subset\left[t_{0},+\infty\right)$.

The notion of stability for a continuos LTI system

$$
\mathscr{L}: \mathscr{I} \rightarrow \mathscr{D}^{\prime}
$$

relies on requests of the following form:

- particular classes of input signals (for instance $L^{\infty}$-signals) must have outputs in particular classes of signals (for instance $L^{\infty}$-signals).

- sequences of input signals converging in a suitable sense (for instance in $L^{\infty}$ ) must have outputs converging in some suitable sense (for instance still in $L^{\infty}$ ).

Notice that the conditions outlined as an example in the above parentheses concern the notion of $L^{\infty}$-stability, often denoted BIBO-stability.

In the following theorems we do not refer to any explicit definition of stability but we give results of the above form, which can be used in the different situations one need to consider.

So, as in the previous sections, let $P(X), Q(X) \in \mathbb{C}[X]$ be the polynomials associated with $P(D), Q(D)$ :

$$
\begin{gathered}
P(X)=c\left(X-s_{1}\right)^{p_{1}} \cdots\left(X-s_{r}\right)^{p_{r}} \\
Q(X)=\left(X-s_{1}\right)^{q_{1}} \cdots\left(X-s_{r}\right)^{q_{r}} Q_{1}(X)
\end{gathered}
$$

let $Q(s) / P(s)$ be the related rational function and let $\Gamma, \Omega$ be the distributions defined in Equations (6) and (7).

The following theorem gives the answers to the problem of causality and stability related to Theorems 3.1, 3.2, 3.3, 3.4.

Theorem 4.1 Let $1 \leqslant p \leqslant \infty$. Assume that $Q(s) / P(s)$ has no pole on the complex imaginary axis (i.e. for every $l$ such that $\sigma_{l}=0$, it is $q_{l} \geqslant p_{l}$ ) and consider the LTI differential system related to $P(D), Q(D)$

$$
\begin{array}{ccc}
\mathscr{L}_{\Gamma}: \mathscr{D}^{\prime}{ }^{p} & \longrightarrow & \mathscr{D}^{\prime} \\
f & \mapsto & \Gamma * f
\end{array}
$$

The following statements hold:

a) $\Gamma \in \mathscr{D}_{L^{1}}^{\prime} \subset \dot{\mathscr{D}}_{L^{\infty}}^{\prime}$

b) $\mathscr{L}_{\Gamma}\left(\mathscr{D}_{L^{p}}^{\prime}\right) \subset \mathscr{D}_{L^{p}}^{\prime}$

c) $\mathscr{L}_{\Gamma}: \mathscr{D}_{L^{p}}^{\prime} \rightarrow \mathscr{D}_{L^{p}}^{\prime}$ is continuous with respect to the strong convergence in both spaces, and with respect to the weak convergence in both spaces

d) $\mathscr{L}_{\Gamma}\left(L^{p}\right) \subset L^{p} \Leftrightarrow \operatorname{deg} Q(D) \leqslant \operatorname{deg} P(D)$

Under this assumption $\mathscr{L}_{\Gamma}: L^{p} \rightarrow L^{p}$ is continuous with respect to the usual norm in both spaces

e) $\mathscr{L}_{\Gamma}: \mathscr{D}_{L^{p}}^{\prime} \rightarrow \mathscr{D}^{\prime}$ (respectively: its restriction to $L^{p}$ ) is causal $\Leftrightarrow Q(s) / P(s)$ has no pole in the complex halfplane $\{s \in \mathbb{C}: \operatorname{Re}(s)>0\}$ (i.e. for every l s.t. $\sigma_{l}>0$ it is $\left.q_{l} \geq p_{l}\right)$ 
Proof. a) See Equations (6)-(8) and relative comments.

b) It follows by [3, Thm. 4.1].

c) It follows by $[3$, Thms. 3.1, 3.3].

d) If $\operatorname{deg} Q(D) \leqslant \operatorname{deg} P(D)$, then $\Gamma(t)=\Gamma_{0}(t)+\Lambda(t)$, where (setting, as in Section 2, $\left.L_{-}=\left\{l: p_{l}>q_{l}, \sigma_{l}<0\right\}, L_{+}=\left\{l: p_{l}>q_{l}, \sigma_{l}>0\right\}\right)$

$$
\begin{aligned}
\Gamma_{0}(t) & =\sum_{l \in L_{-}} \Lambda_{l}(t) e^{s_{l} t} H(t)-\sum_{l \in L_{+}} \Lambda_{l}(t) e^{s_{l} t} H(-t) \in L^{1} \cap L^{\infty} \\
\Lambda(t) & =K \delta(t) \quad \text { with } K \in \mathbb{C}
\end{aligned}
$$

As a consequence, for every $f \in L^{p}$, we have

$$
\mathscr{L}_{\Gamma}(f)=\Gamma_{0} * f+K f
$$

By Young's Theorem we obtain

$$
\begin{aligned}
& \mathscr{L}_{\Gamma}(f)=\Gamma_{0} * f+K f \in L^{p} \\
& \left\|\mathscr{L}_{\Gamma}(f)\right\|_{p}=\left\|\Gamma_{0} * f+K f\right\|_{p} \leqslant\left(\left\|\Gamma_{0}\right\|_{1}+|K|\right)\|f\|_{p}
\end{aligned}
$$

Suppose that $\operatorname{deg} Q(D)=\operatorname{deg} P(D)+\nu$ with $\nu>0$. Then $\Gamma(t)=\Gamma_{0}(t)+\Lambda(t)$ where

$$
\begin{aligned}
& \Gamma_{0}(t)=L^{1} \quad \text { (see above) } \\
& \Lambda(t)=\sum_{h=0}^{\nu} K_{h} \delta^{(h)}(t) \quad \text { with } K_{\nu} \neq 0
\end{aligned}
$$

Let $f(t)=\chi_{(-1,1)}(t) \in L^{p}$, then

$$
\mathscr{L}_{\Gamma}(f(t))=\left(\Gamma_{0} * f\right)(t)+K_{0} f(t)+\sum_{h=1}^{\nu} K_{h}\left(\delta^{(h-1)}(t+1)-\delta^{(h-1)}(t-1)\right)
$$

Since $\left(\Gamma_{0} * f\right)(t)+K_{0} f(t) \in L^{p}$ and $K_{\nu} \neq 0$, we obtain $\mathscr{L}_{\Gamma}(f(t)) \notin L^{p}$.

e) By [3, Thm. 4.6] $\mathscr{L}_{\Gamma}$ is causal if and only if supp $\Gamma \subset[0,+\infty)$; thanks to the definition of $\Gamma(t)$, this happens if and only if there doesn't exist $l$ such that $\sigma_{l}>0$ and $p_{l}>q_{l}$. Obviously, if supp $\Gamma \subset[0,+\infty)$ then the restriction of $\mathscr{L}_{\Gamma}$ to $L^{p}$ is causal. If the restriction of $\mathscr{L}_{\Gamma}$ to $L^{p}$ is causal, write $\delta=\mathscr{E}^{\prime}-\lim _{k \rightarrow \infty} \varphi_{k}$ where $\varphi_{k} \in \mathscr{D}$, supp $\varphi_{k} \subset[0,+\infty)$; by $\left[2\right.$, Theorem 2.1] we obtain $\Gamma=\mathscr{E}^{\prime}-\lim _{k \rightarrow \infty} \mathscr{L}_{\Gamma}\left(\varphi_{k}\right)$; since supp $\mathscr{L}_{\Gamma}\left(\varphi_{k}\right) \subset[0,+\infty)$ then supp $\Gamma \subset[0,+\infty)$.

The following theorem gives the answers to the problem of causality and stability related to Theorem 3.5.

Theorem 4.2 Let $p=1$. Assume that $Q(s) / P(s)$ has no pole of order $>1$ on the complex imaginary axis (i.e. for every $l$ such that $\sigma_{l}=0$, it is $q_{l} \geqslant p_{l}-1$ ), and consider the family (depending on $\mu$ ) of the LTI differential systems

$$
\begin{aligned}
\mathscr{L}_{\mu}=\mathscr{L}_{\Phi_{\mu}}: \mathscr{D}_{L^{1}}^{\prime} & \rightarrow \mathscr{D}^{\prime} \\
f & \mapsto \Phi_{\mu} * f
\end{aligned}
$$

related to $P(D), Q(D)$. The following statements hold:

a) $\Phi_{\mu} \in \mathscr{D}_{L^{\infty}}^{\prime}$

b) $\mathscr{L}_{\mu}\left(\mathscr{D}_{L^{1}}^{\prime}\right) \subset \mathscr{D}_{L^{\infty}}^{\prime}$ 
c) $\mathscr{L}_{\mu}: \mathscr{D}_{L^{1}}^{\prime} \rightarrow \mathscr{D}_{L^{\infty}}^{\prime}$ is continuous with respect to the strong convergence in both spaces

d) $\mathscr{L}_{\mu}\left(L^{1}\right) \subset L^{\infty} \Leftrightarrow \operatorname{deg} Q(D)<\operatorname{deg} P(D)$

Under this assumption $\mathscr{L}_{\mu}: L^{1} \rightarrow L^{\infty}$ is continuous with respect to the usual norms in both spaces

e) In the family $\mathscr{L}_{\mu}: \mathscr{D}_{L^{1}}^{\prime} \rightarrow \mathscr{D}^{\prime}$ (or respectively in the family of their restrictions to $\left.L^{1}\right)$ there exists a causal system $\Leftrightarrow Q(s) / P(s)$ has no pole in the complex halfplane $\{s \in \mathbb{C}: \operatorname{Re}(s)>0\}$

under this assumption $\mathscr{L}_{0}=\mathscr{L}_{(\Gamma+\Omega)}: \mathscr{D}_{L^{1}}^{\prime} \rightarrow \mathscr{D}^{\prime}$ (resp. its restriction to $L^{1}$ ) is the only causal system in the family

Proof. a) Already stated in b) of Theorem 3.5.

b) It follows by [3, Thm. 4.1].

c) It follows by $[3$, Thm. 3.1].

d) If $\operatorname{deg} Q(D)<\operatorname{deg} P(D)$, as in the first part of the Proof of d) of Theorem 4.1, since $\Gamma=\Gamma_{0} \in L^{1} \cap L^{\infty}$ we obtain that $\mathscr{L}_{\mu}\left(L^{1}\right) \subset L^{\infty}$ and that $\mathscr{L}_{\mu}: L^{1} \rightarrow L^{\infty}$ is continuous with respect to the usual norms in both spaces.

If $\operatorname{deg} Q(D)>\operatorname{deg} P(D)$, as in the second part of the Proof of d) of Theorem 4.1, we obtain that $\mathscr{L}_{\mu}\left(\chi_{-1,1}(t)\right) \notin L^{\infty}$.

Finally let $\operatorname{deg} Q(D)=\operatorname{deg} P(D)$, and let $f(t) \in L^{1}$ be such that $f(t) \notin L^{\infty}$. It is easily seen that $\mathscr{L}_{\mu}(f(t)) \notin L^{\infty}$.

e) By [3, Thm. 4.6] $\mathscr{L}_{\mu}$ is causal if and only if supp $\Phi_{\mu} \subset[0,+\infty)$; by the definition of $\Phi_{\mu}$, this happens if an only if the following two conditions are verified: i) $\mu=0$, ii) there doesn't exist $l$ such that $\sigma_{l}>0$ and $p_{l}>q_{l}$. Moreover if supp $\Phi_{\mu} \not \subset[0,+\infty)$, as in Theorem 4.1 it is easily seen that the restriction of $\mathscr{L}_{\mu}$ to $L^{1}$ is not causal.

\section{A Appendix: Uniformly almost periodic functions}

Let $f(t): \mathbb{R} \rightarrow \mathbb{C}$ be a function, and let $\varepsilon \neq 0$. In this Section we call almost period of $f(t)$ relative to $\varepsilon$ a real number $T>0$ such that for every $t_{0} \in \mathbb{R}$, there exists $\tau \in\left[t_{0}, t_{0}+T\right]$ satisfying

$$
|f(t+\tau)-f(t)|<\varepsilon \text { for every } t \in \mathbb{R}
$$

The function $f(t)$ is be called uniformly almost periodic if

- $f(t) \in C^{0}$

- for every $\varepsilon>0$ there exists an almost period $T$ of $f(t)$ relative to $\varepsilon$

The following results are well known (see [1])

- every continuous periodic function is uniformly almost periodic

- the set of uniformly almost periodic functions is a vector subspace of $C^{0}$

- every linear combination of continuous periodic functions is uniformly almost periodic

- if $f(t)$ is uniformly almost periodic then

- $f(t)$ is bounded and uniformly continuous

- Re $f(t), \operatorname{Im} f(t)$ are uniformly almost periodic

- for every $\lambda \in \mathbb{R}, f(\lambda t), f(t+\lambda)$ are uniformly almost periodic 
The following theorem proves that every non null uniformly almost periodic function cannot be null at infinity.

Theorem A.1 Let $f(t)$ be an uniformly almost periodic function; the following statements are equivalent:
a) $\lim _{t \rightarrow+\infty} f(t)=0$
b) $\lim _{t \rightarrow-\infty} f(t)=0$
c) $f(t)=0$ for every $t \in \mathbb{R}$

Proof. a) $\Rightarrow$ c). Let $n>1$. By the assumption there exists a real number $t_{n}>0$ such that

$$
\left|f\left(t_{n}+t\right)\right|<1 / n \text { for every } t>0
$$

Let $T_{n}$ be an almost period of $f(t)$ relative to $1 / n$. Then there exists

$$
\tau_{n} \in\left[-n-t_{n}-T_{n},-n-t_{n}\right]
$$

such that

$$
\left|f\left(t+\tau_{n}\right)-f(t)\right|<1 / n \text { for every } t \in \mathbb{R}
$$

Writing $\tau_{n}=-n-t_{n}-\sigma_{n}$, with $0 \leqslant \sigma_{n} \leqslant T_{n}$, we obtain

$$
\left|f\left(t-n-t_{n}-\sigma_{n}\right)-f(t)\right|<1 / n \text { for every } t \in \mathbb{R}
$$

Substituting $t$ with $t_{n}+t$ we obtain

$$
\left|f\left(-n-\sigma_{n}+t\right)-f\left(t_{n}+t\right)\right|<1 / n \text { for every } t \in \mathbb{R}
$$

and hence

$$
\left|f\left(-n-\sigma_{n}+t\right)\right|<\left|f\left(t_{n}+t\right)\right|+1 / n \text { for every } t \in \mathbb{R}
$$

As a consequence it is

$$
\left|f\left(-n-\sigma_{n}+t\right)\right|<2 / n \text { for every } t>0
$$

and hence

$$
|f(t)|<2 / n \text { for every } t>-n
$$

b) $\Rightarrow \mathrm{c})$. Let $g(t)=f(-t)$ and apply the previous result to $g(t)$.

Obviously $\mathrm{c}) \Rightarrow \mathrm{a}), \mathrm{c}) \Rightarrow \mathrm{b}$ ).

The following theorem proves that every regularization of an uniformly almost periodic function is uniformly almost periodic.

Theorem A.2 Let $f(t)$ be an uniformly almost periodic function, and let $\varphi(t) \in \mathscr{D}$. Then $(f * \varphi)(t)$ is uniformly almost periodic.

Proof. If $\varphi(t)=0$, the statement is obvious. Let $\varphi(t) \neq 0$, and let $\mu=\int|\varphi|>0$.

Let $\varepsilon>0$, let $T>0$ be an almost period of $f(t)$ relative to $\varepsilon /(2 \mu)$, and let $t_{0} \in \mathbb{R}$.

There exists $\tau \in\left[t_{0}, t_{0}+T\right]$ such that

$$
|f(t+\tau)-f(t)|<\varepsilon /(2 \mu) \text { for every } t \in \mathbb{R}
$$


Write $f_{\tau}(t)=f(t+\tau)$. For every $t \in \mathbb{R}$ it is

$$
\begin{aligned}
& |(f * \varphi)(t+\tau)-(f * \varphi)(t)|=\left|\left(f_{\tau} * \varphi\right)(t)-(f * \varphi)(t)\right|= \\
& \quad=\left|\left(\left(f_{\tau}-f\right) * \varphi\right)(t)\right|=\left|\int\left(f_{\tau}-f\right)(x) \varphi(t-x) \mathrm{d} x\right| \leqslant \\
& \quad \leqslant \int|f(x+\tau)-f(x)| \cdot|\varphi(t-x)| \mathrm{d} x \leqslant(\varepsilon /(2 \mu)) \int|\varphi|=\varepsilon / 2<\varepsilon
\end{aligned}
$$

As a consequence $T$ is an almost period of $(f * \varphi)(t)$ relative to $\varepsilon$.

As corollaries of Theorem A.1 and A.2 we can now state the results used in the proofs of the theorems of Sections 2 and 3.

Corollary A.1 Let $\omega_{1}, \ldots, \omega_{\nu} \in \mathbb{R}$ be pairwise distinct, and $c_{1}, \ldots, c_{\nu} \in \mathbb{C}$ be not all equal to 0 . Then there exist $\mu>0$ and two sequences $\tau_{k}^{\prime}, \tau_{k}^{\prime \prime} \in \mathbb{R}$ such that

- $\lim _{k \rightarrow \infty} \tau_{k}^{\prime}=+\infty, \quad\left|\sum_{l=1}^{\nu} c_{l} e^{i \omega_{l} \tau_{k}^{\prime}}\right|>\mu$ for every $k$

- $\lim _{k \rightarrow \infty} \tau_{k}^{\prime \prime}=-\infty, \quad\left|\sum_{l=1}^{\nu} c_{l} e^{i \omega_{l} \tau_{k}^{\prime \prime}}\right|>\mu$ for every $k$

Proof. Simply observe that $\sum_{l=1}^{\nu} c_{l} e^{i \omega_{l} t}$ is uniformly almost periodic (as a linear combination of continuous periodic functions), and non-zero (since the family $e^{i \omega_{l} t}, l=1, \ldots, \nu$ is linearly independent); then apply Theorem A.1.

Corollary A.2 Let $\omega_{1}, \ldots, \omega_{\nu} \in \mathbb{R}$ be pairwise distinct, and let $c_{1}, \ldots, c_{\nu} \in \mathbb{C}$. The following statements are equivalent

a) $\sum_{l=1}^{\nu} c_{l} e^{i \omega_{l} t}$ is a distribution null at $\infty$

b) $\sum_{l=1}^{\nu} c_{l} e^{i \omega_{l} t}$ is a distribution null at $-\infty$

c) $c_{1}=\cdots=c_{\nu}=0$

Proof. Obviously a) $\Rightarrow$ b), and $c) \Rightarrow a$ ).

$\mathrm{b}) \Rightarrow \mathrm{c})$. Let $B \in \mathbb{R}$ be such that

$$
0<B<\min \left\{\frac{\pi}{4 \cdot\left|\omega_{l}\right|}: l \text { such that } \omega_{l} \neq 0\right\}
$$

and let $\varphi(t) \in \mathscr{D}$ be such that $\operatorname{supp} \varphi \subset(-B, B), \varphi(t) \geqslant 0$ for every $t \in \mathbb{R}, \int \varphi \neq 0$. A straightforward argument proves that $\hat{\varphi}\left(\omega_{l}\right) \neq 0$ for every $l$ (here $\hat{\varphi}(\omega)$ denotes the Fourier transform of $\varphi(t))$.

Observe that

$$
\left(\sum_{l=1}^{\nu} c_{l} e^{i \omega_{l} t}\right) * \varphi(t)=\sum_{l=1}^{\nu} c_{l} \hat{\varphi}\left(\omega_{l}\right) e^{i \omega_{l} t}
$$

is a function null at $-\infty$ in the usual sense. As a consequence, by Corollary A.1, for every $l$ we have $c_{l} \hat{\varphi}\left(\omega_{l}\right)=0$, and hence $c_{l}=0$ 
Corollary A.3 Let $\omega_{1}, \ldots, \omega_{\nu} \in \mathbb{R}$ be pairwise distinct, and $M_{1}(t), \ldots, M_{\nu}(t)$ be polynomial functions not all constant. Then there exist two sequences $\tau_{k}^{\prime}, \tau_{k}^{\prime \prime} \in \mathbb{R}$ such that

- $\lim _{k \rightarrow \infty} \tau_{k}^{\prime}=+\infty, \quad \lim _{k \rightarrow \infty}\left|\sum_{l=1}^{\nu} M_{l}\left(\tau_{k}^{\prime}\right) e^{i \omega_{l} \tau_{k}^{\prime}}\right|=+\infty$

- $\lim _{k \rightarrow \infty} \tau_{k}^{\prime \prime}=-\infty, \quad \lim _{k \rightarrow \infty}\left|\sum_{l=1}^{\nu} M_{l}\left(\tau_{k}^{\prime \prime}\right) e^{i \omega_{l} \tau_{k}^{\prime \prime}}\right|=+\infty$

Proof. Write the polynomial functions $M_{l}(t)$ in the form $M_{l}(t)=\sum_{h=0}^{p} m_{l h} t^{h}$, with $p \geqslant 1$ and at least one of the coefficients $m_{1 p}, \ldots, m_{\nu p}$ non null. By Corollary A.1, there exists $\mu>0$ and two sequences $\tau_{k}^{\prime}, \tau_{k}^{\prime \prime} \in \mathbb{R}$ such that

- $\lim _{k \rightarrow \infty} \tau_{k}^{\prime}=+\infty, \quad\left|\sum_{l=1}^{\nu} m_{l p} e^{i \omega_{l} \tau_{k}^{\prime}}\right|>\mu$ for every $k$

- $\lim _{k \rightarrow \infty} \tau_{k}^{\prime \prime}=-\infty, \quad\left|\sum_{l=1}^{\nu} m_{l p} e^{i \omega_{l} \tau_{k}^{\prime \prime}}\right|>\mu$ for every $k$

Then observe that for every $k$ it is

$\sum_{l=1}^{\nu} M_{l}\left(\tau_{k}^{\prime}\right) e^{i \omega_{l} \tau_{k}^{\prime}}=\left(\tau_{k}^{\prime}\right)^{p}\left(\alpha_{k}^{\prime}+\sum_{l=1}^{\nu} m_{l p} e^{i \omega_{l} \tau_{k}^{\prime}}\right), \sum_{l=1}^{\nu} M_{l}\left(\tau_{k}^{\prime \prime}\right) e^{i \omega_{l} \tau_{k}^{\prime \prime}}=\left(\tau_{k}^{\prime \prime}\right)^{p}\left(\alpha_{k}^{\prime \prime}+\sum_{l=1}^{\nu} m_{l p} e^{i \omega_{l} \tau_{k}^{\prime \prime}}\right)$

where $\lim _{k \rightarrow \infty} \alpha_{k}^{\prime}=0$ and $\lim _{k \rightarrow \infty} \alpha_{k}^{\prime \prime}=0$.

Corollary A.4 Let $s_{1}=\sigma_{1}+i \omega_{1}, \ldots, s_{\nu}=\sigma_{\nu}+i \omega_{\nu} \in \mathbb{C}$ be pairwise distinct, $M_{1}(t), \ldots, M_{\nu}(t)$ be polynomial functions not all null, and $P_{0}(t)$ be a polynomial function. If $\sigma_{l} \neq 0$ for every $l=1, \ldots, \nu$ then

a) $\sum_{l=1}^{\nu} M_{l}(t) e^{s_{l} t}+P_{0}(t)$ is not a slowly increasing function in the usual sense

b) $\sum_{l=1}^{\nu} M_{l}(t) e^{s_{l} t} \notin \mathscr{S}^{\prime}$

Proof. Changing, if necessary, the family of indices, we may assume $M_{l}(t)$ not null for every $l$.

a) Assume first that there exists an $l$ s.t. $\sigma_{l}>0$, and let

$$
\sigma=\max \left\{\sigma_{1}, \ldots, \sigma_{\nu}\right\}>0
$$

Then it is

$$
\sum_{l=1}^{\nu} M_{l}(t) e^{s_{l} t}+P_{0}(t)=e^{\sigma t}\left(\alpha(t)+\sum_{l: \sigma_{l}=\sigma} M_{l}(t) e^{i \omega_{l} t}\right)
$$

where $\lim _{t \rightarrow+\infty} \alpha(t)=0$. By Corollaries A.1 and A.3, there exist $\mu>0$ and a sequence $\tau_{k} \in \mathbb{R}$ such that

- $\lim _{k \rightarrow \infty} \tau_{k}=+\infty$ 
- $\left|\sum_{l: \sigma_{l}=\sigma} M_{l}\left(\tau_{k}\right) e^{i \omega_{l} \tau_{k}}\right|>\mu$ for every $k$

As a consequence, $\left|\sum_{l=1}^{\nu} M_{l}(t) e^{s_{l} t}+P_{0}(t)\right|$ cannot be bounded by any $|F(t)|$ where $F(t)$ is a polynomial function.

Assume now that $\sigma_{l}<0$ for every $l$, and let $\Phi(t)=\sum_{l=1}^{\nu} M_{l}(-t) e^{-s_{l} t}+P_{0}(-t)$; by the previous result $\Phi(t)$ is not slowly increasing in the usual sense; as a consequence, also $\Phi(-t)=\sum_{l=1}^{\nu} M_{l}(t) e^{s_{l} t}+P_{0}(t)$ is not.

b) Let $f(t)=\sum_{l=1}^{\nu} M_{l}(t) e^{s_{l} t}$; by [9, Chapter VII, Thm. VI] $f(t) \in \mathscr{S}^{\prime}$ if and only if there exist $n \in \mathbb{N}, g(t) \in C^{0}$ such that

- $g(t)$ is slowly increasing in the usual sense

- $f(t)=g^{(n)}(t)$

It is well known that every $g(t) \in \mathscr{D}^{\prime}$ such that $f(t)=g^{(n)}(t)$ may be written in the form

$$
g(t)=\sum_{l=1}^{\nu} N_{l}(t) e^{s_{l} t}+Q_{0}(t)
$$

where $N_{l}(t)$ and $Q_{0}(t)$ are polynomial functions. Obviously there exists $N_{l}(t) \neq 0$. By a), $g(t)$ cannot be slowly increasing in the usual sense.

Corollary A.5 Let $F(t)=\sum_{l=0}^{p} t^{l} f_{l}(t)$ where $f_{l}(t)$ 's are uniformly almost periodic functions, $p \geqslant 1, f_{p}(t) \neq 0$. Then $F(t), F(t) H(t), F(t) H(-t) \notin \mathscr{D}_{L^{\infty}}^{\prime}$.

Proof. Changing if necessary $F(t)$ with $i F(t)$, we may assume Re $f_{p}(t) \neq 0$. Changing if necessary $F(t)$ with $-F(t)$, by Theorem A.1 we may assume that there exist a real number $\mu>0$ and a sequence $\tau_{k} \in \mathbb{R}$ such that

$$
\lim _{k \rightarrow \infty} \tau_{k}=+\infty, \quad \operatorname{Re} f_{p}\left(\tau_{k}\right)>\mu \text { for every } k
$$

Let $T$ be an almost period of $\operatorname{Re} f_{p}(t)$ relative to $\mu / 8$.

For every $\tau_{k}$, there exists a real number $-\tau_{k}+\sigma_{k} \in\left[-\tau_{k},-\tau_{k}+T\right]$ (i.e. a real number $\left.\sigma_{k} \in[0, T]\right)$ such that

$$
\left|\operatorname{Re} f_{p}\left(t-\tau_{k}+\sigma_{k}\right)-\operatorname{Re} f_{p}(t)\right|<\mu / 8 \quad \text { for every } t \in \mathbb{R}
$$

and hence, changing $t$ with $t+\tau_{k}$, such that

$$
\left|\operatorname{Re} f_{p}\left(t+\sigma_{k}\right)-\operatorname{Re} f_{p}\left(t+\tau_{k}\right)\right|<\mu / 8 \quad \text { for every } t \in \mathbb{R}
$$

Since every $\sigma_{k} \in[0, T]$, changing if necessary the sequence $\tau_{k}$ with a subsequence, we may assume that there exists $\sigma \in[0, T]$ such that $\lim _{k \rightarrow \infty} \sigma_{k}=\sigma$.

Since $\operatorname{Re} f_{p}(t)$ is uniformly continuous, there exists a real number $\zeta>0$ such that for every pair $\left(t^{\prime}, t^{\prime \prime}\right)$ such that $\left|t^{\prime \prime}-t^{\prime}\right|<\zeta$ it is $\left|\operatorname{Re} f_{p}\left(t^{\prime \prime}\right)-\operatorname{Re} f_{p}\left(t^{\prime}\right)\right|<\mu / 8$. Changing if 
necessary the sequence $\tau_{k}$ with a subsequence, we may assume that $\left|\sigma_{k}-\sigma\right|<\zeta$ for every $\tau_{k}$, and hence

$$
\left|\operatorname{Re} f_{p}\left(t+\sigma_{k}\right)-\operatorname{Re} f_{p}(t+\sigma)\right|<\mu / 8 \quad \text { for every } t \in \mathbb{R}
$$

As a consequence of Equations (A.1) and (A.2) it is

$$
\begin{aligned}
& \left|\operatorname{Re} f_{p}(t+\sigma)-\operatorname{Re} f_{p}\left(t+\tau_{k}\right)\right|= \\
& \quad=\left|\operatorname{Re} f_{p}(t+\sigma)-\operatorname{Re} f_{p}\left(t+\sigma_{k}\right)+\operatorname{Re} f_{p}\left(t+\sigma_{k}\right)-\operatorname{Re} f_{p}\left(t+\tau_{k}\right)\right| \\
& \quad<2 \cdot \mu / 8=\mu / 4 \text { for every } t \in \mathbb{R}
\end{aligned}
$$

Taking $t=0$ in Equation (A.3) we obtain $\left|\operatorname{Re} f_{p}(\sigma)-\operatorname{Re} f_{p}\left(\tau_{k}\right)\right|<\mu / 4$. Since $\operatorname{Re} f_{p}\left(\tau_{k}\right)>\mu$, it is $\operatorname{Re} f_{p}(\sigma)>\mu-\mu / 4=3 \mu / 4$. Then there exists a real number $B>0$ such that

$$
\operatorname{Re} f_{p}(t+\sigma)>3 \mu / 4 \text { for every } t \in(-B, B)
$$

As a consequence of Equation (A.3) it is also

$$
\begin{array}{r}
\operatorname{Re} f_{p}\left(t+\tau_{k}\right)>3 \mu / 4-\mu / 4=\mu / 2 \\
\quad \text { for every } t \in(-B, B)
\end{array}
$$

Last, observe that, for every $t \neq 0, F(t)$ may be uniquely written in the form

$$
F(t)=t^{p}\left(\psi(t)+f_{p}(t)\right)
$$

with $\psi(t)$ continuous on $\mathbb{R} \backslash\{0\}$, and such that there exists a real number $C>0$ s.t.

$$
|\psi(t)|<\mu / 4 \text { for every }|t|>C
$$

Let $\Phi(t)=F(t) H(t)$.

Let $\varphi(t) \in \mathscr{D}$ be such that supp $\varphi \subset(-B, B), \varphi(t) \geqslant 0$ for every $t \in \mathbb{R}, \int \varphi=1$. For every $\tau_{k}>C+B$ it is

$$
\begin{aligned}
\operatorname{Re} & (\Phi * \varphi)\left(\tau_{k}\right)= \\
& =\int_{\tau_{k}-B}^{\tau_{k}+B} t^{p}\left(\operatorname{Re} \psi(t)+\operatorname{Re} f_{p}(t)\right) \varphi\left(\tau_{k}-t\right) \mathrm{d} t= \\
& =\int_{-B}^{B}\left(\tau_{k}+t\right)^{p}\left(\operatorname{Re} \psi\left(\tau_{k}+t\right)+\operatorname{Re} f_{p}\left(\tau_{k}+t\right)\right) \varphi(-t) \mathrm{d} t
\end{aligned}
$$

Since for every $t \in(-B, B)$ it is

- $\left(\tau_{k}+t\right)^{p} \geqslant\left(\tau_{k}-B\right)^{p}>0$

- $\operatorname{since} \tau_{k}+t>C, \operatorname{Re} \psi\left(\tau_{k}+t\right)+\operatorname{Re} f_{p}\left(\tau_{k}+t\right)>-\mu / 4+\mu / 2=\mu / 4>0$

- $\varphi(-t) \geqslant 0$

then

$$
\operatorname{Re}(\Phi * \varphi)\left(\tau_{k}\right) \geqslant\left(\tau_{k}-B\right)^{p} \cdot(\mu / 4) \cdot \int \varphi=(\mu / 4) \cdot\left(\tau_{k}-B\right)^{p}
$$

As a consequence $(\Phi * \varphi)(t)$ is not bounded in $\mathbb{R}$, and hence $\Phi(t)=F(t) H(t) \notin \mathscr{D}_{L^{\infty}}^{\prime}$.

The proof for $F(t)$ and $F(t) H(-t)$ are straightforward consequences.

Corollary A.6 Let $F(t)=\sum_{l=0}^{p} t^{l} f_{l}(t), \quad G(t)=\sum_{l=0}^{q} t^{l} g_{l}(t)$ where $f_{l}(t)$ 's,$g_{l}(t)$ 's are uniformly almost periodic function. The following statements are equivalent: 
a) $F(t) H(t)+G(t) \in \mathscr{D}_{L^{\infty}}^{\prime}$

b) for every $l \neq 0$ it is $f_{l}(t)=0, g_{l}(t)=0$

Proof. a) $\Rightarrow$ b). Assume for a contradiction that $g_{l}(t) \neq 0$ for some $l \neq 0$. The argument adopted in the Proof of Corollary A.5 may be used to prove that $F(t) H(t)+G(t) \notin \mathscr{D}_{L^{\infty}}$ (simply use a sequence $\tau_{k}$ such that $\lim _{k \rightarrow \infty} \tau_{k}=-\infty$ ) which is absurd. Hence it is $g_{l}(t)=0$ for every $l \neq 0$. In particular $G(t)=g_{0}(t)$ is an uniformly almost periodic function, and hence $G(t) \in \mathscr{D}^{\prime}{ }^{\infty}$.

As a consequence $F(t) H(t) \in \mathscr{D}_{L^{\infty}}^{\prime}$, and hence by Corollary A.5 it is $f_{l}(t)=0$ for every $l \neq 0$.

b) $\Rightarrow$ a). $F(t) H(t)+G(t)$ is a locally integrable bounded function; hence $F(t) H(t)+G(t) \in$ $\mathscr{D}_{L^{\infty}}^{\prime}$.

Corollary A.7 Let $F(t), G(t)$ be uniformly almost periodic functions. If $F(t) H(t)+G(t) \in$ $\dot{\mathscr{D}}_{L^{\infty}}^{\prime}$, then $F(t)=G(t)=0$.

Proof. Let $\Phi(t)=F(t) H(t)+G(t), \varphi(t) \in \mathscr{D}$, and $\widetilde{\varphi}(t)=\varphi(-t)$. By the argument pointed out in the last paragraph of $\left[9\right.$, Chapter VI, section 8] it is $\lim _{|t| \rightarrow \infty}(\Phi * \widetilde{\varphi})(t)=0$. Obviously there exists $t_{0}<0$ such that for every $t<t_{0}$ it is $(\Phi * \widetilde{\varphi})(t)=(G * \widetilde{\varphi})(t)$, hence $\lim _{t \rightarrow-\infty}(G * \widetilde{\varphi})(t)=0$.

By Theorem A.2, $(G * \widetilde{\varphi})(t)$ is uniformly almost periodic, hence by Theorem A.1, $(G *$ $\widetilde{\varphi})(t)=0$ for every $t \in \mathbb{R}$; in particular

$$
\langle G(t), \varphi(t)\rangle=\int G(\tau) \widetilde{\varphi}(0-\tau) \mathrm{d} \tau=(G * \widetilde{\varphi})(0)=0
$$

Hence for every $\varphi \in \mathscr{D}$ it is $\langle G(t), \varphi(t)\rangle=0$. As a consequence $G(t)=0$ and $\Phi(t)=$ $F(t) H(t)$. Let again $\varphi(t) \in \mathscr{D}$, and $\widetilde{\varphi}(t)=\varphi(-t)$. Observe that there exists $t_{0}>0$ such that for every $t>t_{0}$ it is $(\Phi * \widetilde{\varphi})(t)=(F * \widetilde{\varphi})(t)$, hence $\lim _{t \rightarrow+\infty}(F * \widetilde{\varphi})(t)=0$. As a consequence, the argument previously applied to $G(t)$ proves that $F(t)=0$.

\section{References}

[1] A.S. Besicovitch, Almost periodic functions, Cambridge University press, 1932.

[2] M. Ciampa, M. Franciosi, M. Poletti, A note on impulse response for continuous, linear, time-invariant, continuous-time systems, IEEE Trans. Circuits Syst. I, 53, 2006.

[3] M. Ciampa, M. Franciosi, M. Poletti, Continuous LTI systems defined on $L^{p}$ functions and $\mathscr{D}_{L^{p}}^{\prime}$ distributions: analysis by impulse response and convolution, to appear on IEEE Trans. Circuits Syst. I.

[4] J.W. Polderman, J. C. Willems, Introduction to Mathematical Systems Theory: a behavioral approach, Springer-Verlag NY, 1998.

[5] I. Sandberg, Continuous-time linear systems: folklore and fact, Circuits Systems Signal Processing, 21, 337-343, 2002.

[6] - Causality and the impulse response scandal, IEEE Trans. Circuits Syst. I, 50, 810-811, June 2003.

[7] — Bounded inputs and the representation of linear system maps, Circuits, Systems Signal Processing, 24, 103-115, 2005. 
[8] — Continuous multidimensional systems and the impulse response scandal, Multidim. Syst. Signal Processing, 15, 295-299, 2004.

[9] L. Schwartz, Théorie des distributions. Paris: Hermann, 1966.

[10] H.L. Trentelman, J. C. Willems, The Behavioral Approach as a Paradigm for Modelling Interconnected Systems, European Journal of Control, 9, 296-306, 2003. 